\title{
Design Coverage Optimization Based on Position of Constellations and Cost of the Launch Vehicle
}

\author{
SAEID KOHANI ${ }^{1},{ }^{2}$ PENG ZONG, ${ }^{3}$ FENGFAN YANG \\ ${ }^{1}$ Nanjing University of Aeronautics and Astronautics, Astronautics College, Nanjing, JiangSu, \\ 210016, China \\ ${ }^{2}$ Nanjing University of Aeronautics and Astronautics, Astronautics College, Nanjing, JiangSu, \\ 210016, CHINA \\ ${ }^{3}$ College of Electronic and Information Engineering Nanjing University of Aeronautics and \\ Astronautics, Nanjing, JiangSu, 210016, CHINA
}

\begin{abstract}
This research will analyze the tradeoffs between coverage optimization based on Position dilution of precision (PDOP) and cost of the launch vehicle. It adopts MATLAB and STK tools along with multiple objective genetic algorithms (MOGA) to explore the trade space for the constellation designs at different orbital altitudes. The objective of optimal design solutions is inferred to determine the economic and efficient LEO, MEO, HEO or hybrid constellations and simulation results are presented to optimize the design of satellite constellations. The benefits of this research are the optimization of satellite constellation design, which reduces costs and increases regional and global coverage with the least number of satellites. The result of this project is the optimization of the number of constellation satellites in several orbital planes in LEO orbit. Validations are based on reviewing the results of several simulations. The results of graphs and tables are presented in the last two sections and are taken from the results of several simulations.
\end{abstract}

Key Words: Satellite, Orbit, Cost, PDOP, MOGA, Constellation.

Received: April 22, 2021. Revised: October 5, 2021. Accepted: October 29, 2021. Published: November 16, 2021.

\section{Introduction}

The low-Earth orbit (LEO) economy broadly refers to in-space all kinds of applications and activities in LEO, associating to not only satellite design, constellation deployment, launching but more others. Typical missions include:

- Tourism: Commercial human spaceflight and accommodation for tourism and related purposes.

- Research: Basic and applied research in the space environment and microgravity.

- Demo: Technology test and demonstration, qualification, and certification of systems in space.
- Media: Entertainment, media broadcast, and public relations applications.

- Education: Space activities with the purpose of education at any level.

- Manufacturing: Production, typically in large scale or in space environment.

- Satellite deployment: Vehicle or platform based deployment of satellites.

- In-Space transportation: On-orbit maneuvering vehicles, orbital transfer, and the use of platforms as transportation nodes.

- Imaging capture: Earth remote sensing and imaging of in space assets. 
- Resource acquisition: Sample return, mining, and situ resource utilization.

- Satellite service: Satellite servicing with different types of robotic or human repair and maintenance of in-space assets.

- Launch: Earth to space launch or Earth to Earth (typically referred to as 'point to point' transportation).

LEO programs have historically been dominated by government, with less exception of commercial applications. The LEO economy has recently seen increased commercial activity and interest, including commercial human space flight.

This research characterizes the state of LEO economy in terms of investment, demand, and supply. All three are necessary economic building blocks. If supply and demand meet on the price, economy can be flourishing. In the developing of LEO economy, we could see new products and services in a wide range of markets. The emerging LEO economy includes investments that can be characterized as advocacy investment, strategic investment, or financial investment. These kinds of investments can be sequential or level that varies across the market in LEO economy.

\section{PDOP Function}

The batch Pareto fronts produced in this research depends on several calculations. The PDOP (Position solution Dilution of Precision) is calculated by STK in an adequate metric for the scenario. When STK calculates PDOP, it only offers users a few value options: minimum, maximum, average, or percentage to capture the worst cases, the maximum values are determined for each global point over the simulation interval. The global PDOP value is analyzed, on the basis of single PDOP value for grid. Therefore, the median of the maximum values of each grid forms a PDOP metric. The median is used to measure the central tendency because the data distribution at lower altitudes is positively skewed. Figure 1 illustrates the probability density function (PDF) of PDOP in LEO case. The median give a more accurate measure of the central tendency. Figure 2 and Figure 3 illustrate the probability density function for PDOP in MEO and GEO cases respectively. These figures demonstrate the median and mean values are either the same or very similar. Therefore, for those cases, either measure would demonstrate the central tendency of the data. The median value was chosen as PDOP because of its ability to show the central tendency for each of the three cases.

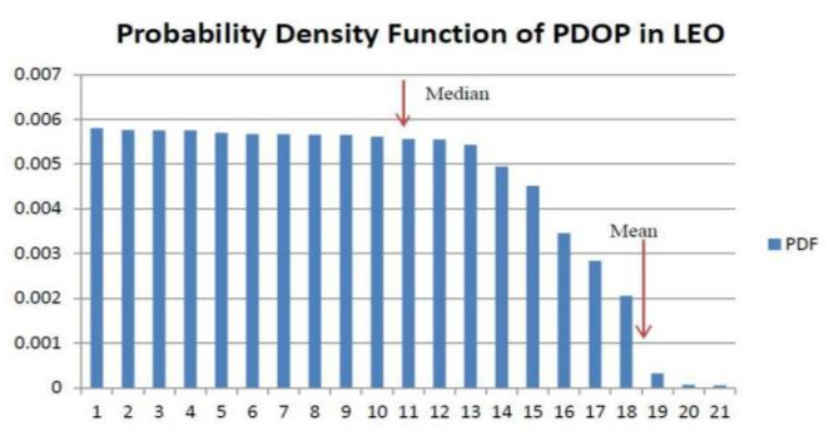

Figure 1: Probability Density Function of PDOP in LEO

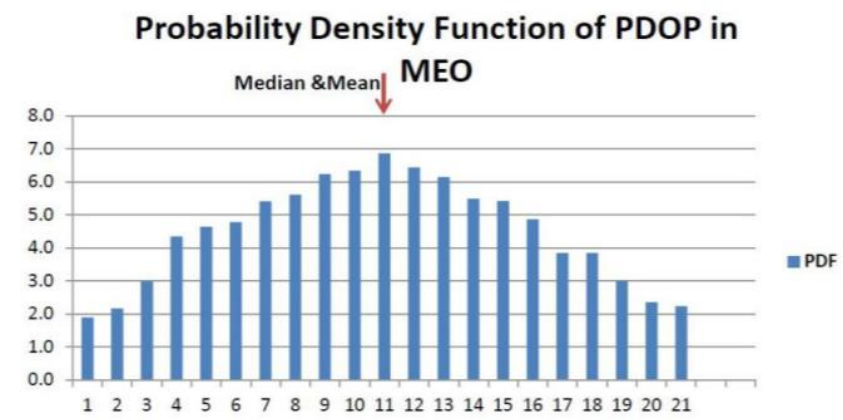

Figure 2: Probability Density Function of PDOP in MEO 


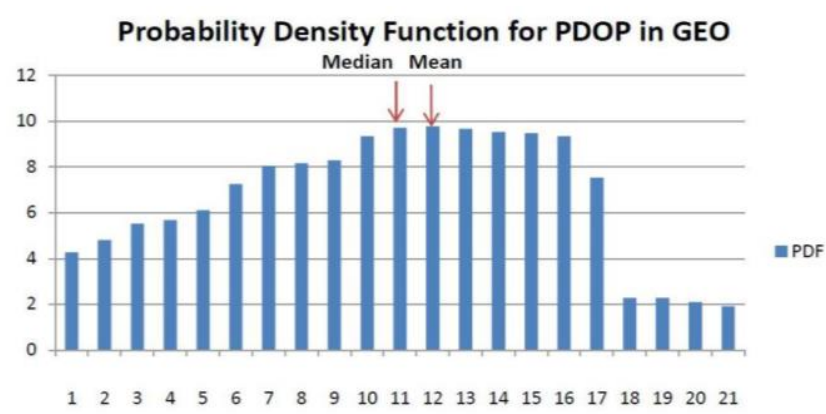

Figure 3: Probability Density Function of PDOP in GEO

It is assumed that the coverage grid in PDOP calculation is sufficiently dense. There must be a balance between resolution and time. Better resolution produces more accurate results, but needs more computation time. The PDOP calculation assumed no terrain limits, which are necessarily reality that cannot be directly reached. Real world includes skyscrapers and mountains that affect the visible of satellites to user on the ground.

In the cost model of objective function, the costs are computed in fiscal year 2010 dollars (FY2010\$). The cost models are only approximations because no model is perfect. It is hereby assumed that a communications payload is an appropriate representation of the constellation satellite. When determining launch vehicle cost, the average launch costs in [1] are applied, even if the costs are separated into LEO and GEO. Therefore, it is assumed that any satellite at an altitude higher than $2000 \mathrm{~km}$ will be launched by GEO vehicles. The current available launch vehicles are Pegasus XL, Minotaur IV, Falcon 9, Atlas 5, and Delta 4 Heavy. All orbits analyzed in this research are assumed to be circular. It means that the power and gain values of one satellite in the link margin calculations are applicable for all satellites. Table 1 summarizes the specifications of GPS satellite at three different elevation angles. The range and path loss are calculated on the satellite altitude. For each test case, a required transmitting power is calculated and needs a small range variety in altitude in order for the MOGA to function properly. Using the assumptions and parameters defined above, the objective functions can be programmed in simulation. This paper outlines the programming details of the objective functions. Each objective function is programmed in separate MATLAB files. The wrapper function and fitness function are defined as the concatenation of objective functions. The function module that calculates PDOP is called by "PDOP.m", and "cost.m" is the function module that determines cost. The PDOP function module using the design vector $\boldsymbol{X}$ outputs a global value of PDOP for the optimization design. STK can use the Connect commands within this function model, [3] To improve computation time, the visibility in STK is set to zero, so the program does not display all time if the PDOP function is called, whilst it runs in background.

Table 1: Gain and Power Specifications for GPS [2]

\begin{tabular}{|l|c|c|c|}
\hline & $\begin{array}{c}\text { Satellite } \\
\text { at } 5^{\circ} \\
\text { Elevation }\end{array}$ & $\begin{array}{c}\text { Satellite } \\
\text { at } 40^{\circ} \\
\text { Elevation }\end{array}$ & $\begin{array}{c}\text { Satellite } \\
\text { at 90 } \\
\text { Elevation }\end{array}$ \\
\hline Range(km) & 25240 & 22020 & 21190 \\
\hline $\begin{array}{l}\text { Satellite } \\
\text { Antenna Gain, } \\
\text { dB }\end{array}$ & 12.1 & 12.9 & 10.2 \\
\hline $\begin{array}{l}\text { Effective } \\
\text { Isotropic } \\
\text { Radiated } \\
\text { Power, dBW }\end{array}$ & 26.4 & 27.2 & 24.5 \\
\hline Path Loss, dB & -159 & -157.8 & -157.1 \\
\hline $\begin{array}{l}\text { Atmospheric } \\
\text { Loss, dB }\end{array}$ & 0.5 & 0.5 & 0.5 \\
\hline $\begin{array}{l}\text { Received Power } \\
\text { Density, } \\
\text { dBW/m^2 }\end{array}$ & -133.1 & -131.1 & -133.1 \\
\hline $\begin{array}{l}\text { Effective Area } \\
\text { of an } \\
\text { Omnidirectional } \\
\text { Antenna, dBm² }\end{array}$ & 25.4 & 25.4 & 25.4 \\
\hline Receive Power & -158.5 & -156.5 & -158.5 \\
\hline
\end{tabular}




\begin{tabular}{|l|c|c|c|}
\hline $\begin{array}{l}\text { Available from } \\
\text { an Isotropic } \\
\text { Antenna, dBW }\end{array}$ & & & \\
\hline $\begin{array}{l}\text { Gain of a } \\
\text { Typical Patch } \\
\text { Receive }\end{array}$ & -4 & 2 & 4 \\
Antenna, dBic & & & \\
\hline $\begin{array}{l}\text { C/A Code } \\
\text { Received Power } \\
\text { Available to a } \\
\text { Typical Receive } \\
\text { Antenna, dBW }\end{array}$ & -162.5 & -154.5 & -154.5 \\
\hline
\end{tabular}

A STK scenario is created by the function file (navigation.m) that defines the simulation time and the location to save the scenario file. The user must ensure that the PDOP file path location (PDOP.m) matches the function file path specified in (navigation.m). In this simulation model of STK, a satellite constellation object is created and named by NAVcon. As mentioned Walker constellations are analyzed in this simulation, the seed of simulation is generated and called NAVSat. Using the seed of satellite, Walker constellations are generated by the specific Walker parameters in $\boldsymbol{X}$ vector. To analyze the coverage of constellation, a definition of object is called as coverage Performance. The coverage of the constellation NAVcon is measured by FOM (figure of merit), which presents PDOP (Position solution Dilution of Precision). In STK, the PDOP values are saved in a matrix as formation of following columns: latitude, minimum, maximum, average, standard deviation, count, and sum. The maximum values are selected to analyze the worst case scenario for the specific case. Lastly, the median of the column of maximum values is used as the final value of PDOP. The cost function consists of the launch vehicle cost, USCM8 NRE, USCM8 recurring, and SSCM NRE. Equation 1 represents the total cost.

$$
\begin{aligned}
\text { Total Cost }= & \text { Cost }_{\text {LV } / \text { Plane }} * N_{\text {planes }}+\text { Cost }_{N R E} \\
& * N_{\text {dev }}+\operatorname{Cost}_{\text {Recurring }} * N_{\text {prod }} \\
& +\operatorname{Cost}_{S S C M} * N_{\text {prod }}
\end{aligned}
$$

where

$$
\begin{gathered}
N_{\text {Planes }}=\text { number of orbital planes } \\
N_{\text {prod }}=\text { total number of satellites } \\
N_{\text {dev }}=1 \text { when } N_{\text {prod }}>0
\end{gathered}
$$

\section{Launch Vehicle Cost Function.}

This paper details the calculations for launch vehicle cost. Before the launch cost can be calculated, the spacecraft mass must be determined. The spacecraft mass is calculated on the mass of payload. To develop a relationship between transmit power and payload mass, a second order polynomial trend is created by using transmit power and the payload mass of such as GPS [4], GLONASS [5], Galileo [6], and Beidou [6].

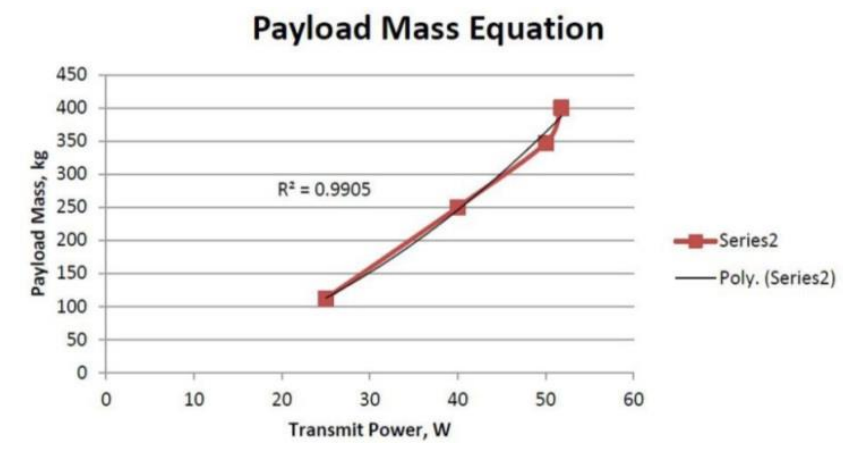

Figure 4: Payload Mass Equation

Table 2 illustrates the specific values. Figure 4 shows the trend of the data. Equation 2 represents the payload mass equation generated from the trendline in Figure 4.

Table 2: Mass and Power for Navigation Constellations

\section{Transmit Payload}




\begin{tabular}{lcc}
\hline & Power, W & Mass, kg \\
\hline Beidou & 52 & 400 \\
GPS & 50 & 347 \\
Glonass & 40 & 250 \\
Galileo & 25 & 112 \\
\hline
\end{tabular}

mass $_{\text {payload }}=0.116 *$ power $_{\text {tx }}^{2}+1.279 *$

power $_{\text {tx }}+8.395$

See Appendix E for detailed equations and calculations of estimating the payload mass and transmit power for GPS, GLONASS and Beidou. Equation 3 is derived from the cost model SMESMAD that represents the spacecraft net mass.

Spacecraft $_{\mathrm{dry}_{\text {mass }}}=$ mass $_{\text {payload }} / 0.32$

The total spacecraft mass is then calculated using Equation 4.

$$
\begin{aligned}
& \text { Spacecraft }_{\text {mass }}=\text { mass }_{\text {payload }}+ \\
& \text { Spacecraft }_{\mathrm{dry}_{\text {mass }}}
\end{aligned}
$$

The cost function acts as the design vector $\boldsymbol{X}$ saved in a structure file called lv. The lv structure defines the different characteristics of the launch vehicles used in the simulation. All launch vehicle values are referenced from [1]. The structure consists of two fields: mass and cost. The mass field possesses two fields: bounds and alt. The bounds field is a matrix of the launch capacities for each vehicle at LEO and GEO. The alt field is data range from $2000 \mathrm{~km}$ to infinity. This field is used to determine launch capacity values on the basis of altitude scenarios.

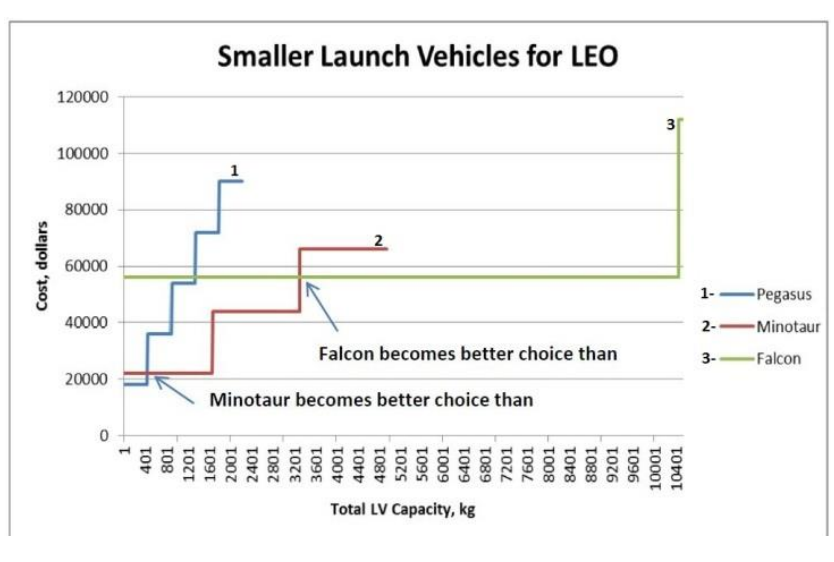

Figure 5: Small Launch Vehicles for LEO

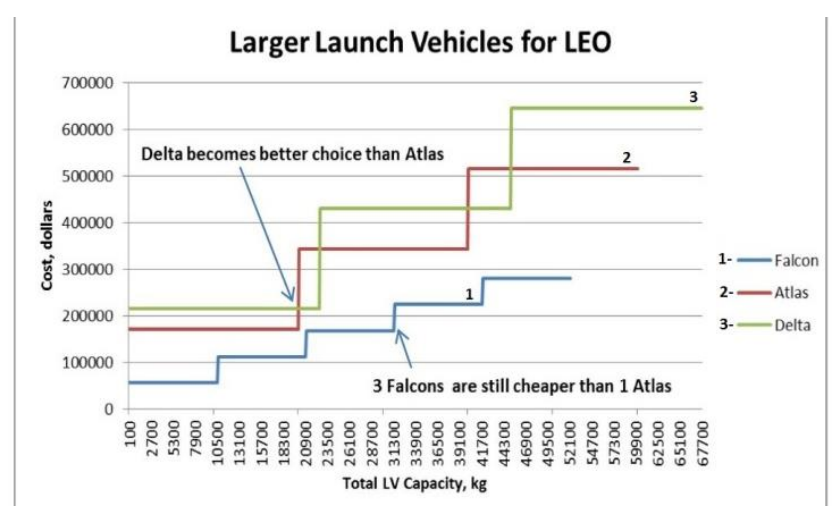

Figure 6: Larger Launch Vehicles for LEO

The cost field is made up of average and efficiency. The average field is a matrix of the average launch cost for different vehicles, which includes cost of the launch vehicle and related launch services [1]. The efficiency is a matrix of the cost efficiencies for LEO and GEO, which is measured as the cost per kilogram placed into orbit. The spacecraft mass determines type, times and cost of the launch vehicle. When the scenario is using LEO, the choices of launch vehicle include Pegasus, Minotaur, Falcon 9, Atlas V, and Delta4H. Figure 5 and 6 illustrate the capacity and cost against the number of launch vehicles. The figure shows that certain launch vehicles may be more beneficial to use than others. When the scenario is using GEO, the available vehicles are Falcon 9, Atlas V, and 
Delta 4H. Figure 7 demonstrates that three Falcon 9 are cheaper than one Atlas V or Delta $4 \mathrm{H}$ in price.

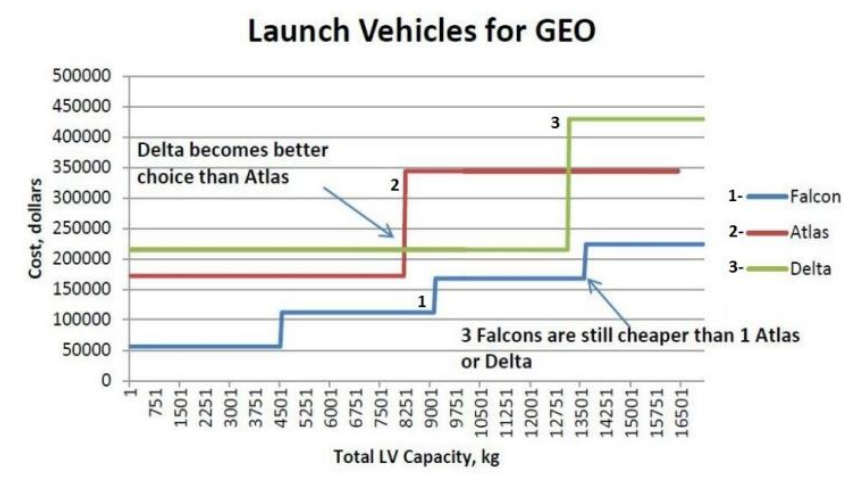

Figure 7: Launch Vehicles for GEO

Addition to the spacecraft mass, number of launch vehicles, $n$, and the launch cost are determined by the orbital plane. Total launch cost is equal to single cost of one orbital plane multiplied by the number of planes. Equation 5 represents the launch cost per plane, Cost $_{L V} /$ Plane.

$$
\begin{aligned}
& \frac{\operatorname{Cost}_{L V}}{\text { Plane }}= \\
& {\left[n_{\text {Pegasus }}, n_{\text {Minotaur }}, n_{\text {Falcon }}, n_{\text {Atlas }}, n_{\text {delta }}\right] *} \\
& {\left[\begin{array}{c}
\text { Cost }_{\text {Pegasus }} \\
\text { ost }_{\text {Minotaur }} \\
\text { Cost }_{\text {Falcon }} \\
\text { Cost }_{\text {Atlas }} \\
\text { Cost }_{\text {Delta }}
\end{array}\right]}
\end{aligned}
$$

The Falcon Heavy is a partially reusable heavy-lift launch vehicle designed and manufactured by SpaceX. It is derived from the Falcon 9 vehicle and consists of a strengthened Falcon 9 first stage as the center core with two additional Falcon 9-like first stages as strap-on boosters.[7] The Falcon Heavy has the largest payload capacity of any currently operational launch vehicles, and the third-largest capacity trailing the Saturn V and Energia. SpaceX conducted the Falcon Heavy's maiden launch on February 6, 2018, at 3:45 p.m. EST (20:45 UTC).[8] The rocket of SpaceX carried a Roadster made by
Tesla whose founder is also Elon Musk to,the space with a dummy dubbed "Starman" as a dummy payload.[9] The second Falcon Heavy launched on April 11, 2019 and all three booster rockets successfully returned to Earth.[10] The third Falcon Heavy successfully launched on June 25, 2019. Since then, the Falcon Heavy has been certified for the National Security Space Launch program.[11]

The Falcon Heavy was designed to be able to carry human into space beyond near Earth orbit, and will be replaced by the more powerful launch system "Starship".[12] Falcon Heavy consists of a structurally strengthened Falcon 9 as the "core" component, with two additional Falcon 9 first stages acting as liquid fuel strap-on boosters,[7] which is conceptually similar to EELV Delta IV Heavy launcher, Atlas V Heavy and Russian Angara A5V. Falcon Heavy has more lift capability than any other operational rocket, with a payload of 63,800 kilograms $(140,700 \mathrm{lb})$ to low Earth orbit, 26,700 kilograms $(58,860 \mathrm{lb})$ to Geostationary Transfer Orbit, and $16,800 \mathrm{~kg}(37,000 \mathrm{lb})$ to trans-Mars injection.[13] The rocket was designed to meet or exceed all requirements of current rating. The structural safety margins are $40 \%$ above flight loads, and higher than $25 \%$ of other rockets.[14]

The first stage of Falcon Heavy is powered by three Falcon 9 derived cores, each equipped with nine Merlin 1D engines. The Falcon Heavy has a total sea-level thrust liftoff of $22,819 \mathrm{kN}(5,130,000 \mathrm{lbf})$ from 27 Merlin 1D engines, while thrust rises to $24,681 \mathrm{kN}(5,549,000 \mathrm{lbf})$ for the craft climbing out of the atmosphere.[15] The rocket upper stage is powered by a single Merlin 1D engine modified for vacuum operation, in which the thrust is $934 \mathrm{kN}$ $(210,000 \mathrm{lbf})$ with an expansion ratio of $117: 1$ and a 
nominal burn time of 397 seconds. During launch, the center core throttles up full power jn a few seconds for additional thrust. This allows a longer burn time. After the side boosters separating, the center core throttles up to maximum thrust again. For additional reliability of restart, the engine has dual redundant pyrophoric igniters (TEA-TEB).[7]
The intersection jointing the upper stage and lower stage of Falcon 9 is a carbon fiber and aluminum composite structure. Stages are detached by reusable separation collets and pneumatic pusher system. 
TABLE 3

Specifications of Falcon Heavy

\begin{tabular}{|c|c|c|c|}
\hline \multirow[t]{2}{*}{ Characteristic } & First stage core unit & \multirow[t]{2}{*}{ Second stage } & \multirow[t]{2}{*}{ Payload fairing } \\
\hline & $(1 \times$ center, $2 \times$ booster $)$ & & \\
\hline Height[66] & $42.6 \mathrm{~m}(140 \mathrm{ft})$ & $12.6 \mathrm{~m}(41 \mathrm{ft})$ & $13.2 \mathrm{~m} \mathrm{(43 \textrm {ft } )}$ \\
\hline Diameter[66] & $3.66 \mathrm{~m}(12.0 \mathrm{ft})$ & $3.66 \mathrm{~m}(12.0 \mathrm{ft})$ & $5.2 \mathrm{~m}(17 \mathrm{ft})$ \\
\hline Dry Mass[66] & $22,200 \mathrm{~kg}(48,900 \mathrm{lb})$ & $4,000 \mathrm{~kg}(8,800 \mathrm{lb})$ & $1,700 \mathrm{~kg}(3,700 \mathrm{lb})$ \\
\hline Fueled mass & $433,100 \mathrm{~kg}(954,800 \mathrm{lb})$ & $111,500 \mathrm{~kg}(245,800 \mathrm{lb})$ & $\mathrm{N} / \mathrm{A}$ \\
\hline \multirow[t]{2}{*}{ Structure type } & LOX tank: monocoque & LOX tank: monocoque & \multirow[t]{2}{*}{ Monocoque halves } \\
\hline & Fuel tank: skin and stringer & Fuel tank: skin and stringer & \\
\hline Structure material & $\begin{array}{l}\text { Aluminum-lithium skin; } \\
\text { aluminum domes }\end{array}$ & $\begin{array}{l}\text { Aluminum-lithium skin; } \\
\text { aluminum domes }\end{array}$ & Carbon fiber \\
\hline Engines & $9 \times$ Merlin 1D & $1 \times$ Merlin 1D Vacuum & \multirow[t]{16}{*}{$\mathrm{N} / \mathrm{A}$} \\
\hline Engine type & Liquid, gas generator & Liquid, gas generator & \\
\hline Propellant & $\begin{array}{l}\text { Subcooled liquid oxygen, } \\
\text { kerosene (RP-1) }\end{array}$ & $\begin{array}{l}\text { Liquid oxygen, kerosene (RP- } \\
\text { 1) }\end{array}$ & \\
\hline $\begin{array}{l}\text { Liquid oxygen tank } \\
\text { capacity[66] }\end{array}$ & $287,400 \mathrm{~kg}(633,600 \mathrm{lb})$ & $75,200 \mathrm{~kg}(165,800 \mathrm{lb})$ & \\
\hline Kerosene tank capacity[66] & $123,500 \mathrm{~kg}(272,300 \mathrm{lb})$ & $32,300 \mathrm{~kg}(71,200 \mathrm{lb})$ & \\
\hline Engine nozzle & Gimbaled, 16:1 expansion & Gimbaled, 165:1 expansion & \\
\hline $\begin{array}{l}\text { Engine } \\
\text { designer/manufacture } \\
\mathbf{r}\end{array}$ & SpaceX & SpaceX & \\
\hline Thrust, stage total & $\begin{array}{l}22,819 \mathrm{kN}\left(5,130,000 \mathrm{lb}_{\mathrm{f}}\right), \text { sea } \\
\text { level }\end{array}$ & $934 \mathrm{kN}\left(210,000 \mathrm{lb}_{\mathrm{f}}\right)$, vacuum & \\
\hline $\begin{array}{l}\text { Propellant feed } \\
\text { system }\end{array}$ & Turbopump & Turbopump & \\
\hline Throttle capability & $\begin{array}{l}\text { Yes: } 816-419 \mathrm{kN}(190,000- \\
108,300 \mathrm{lbf}) \text {, sea level }\end{array}$ & $\begin{array}{l}\text { Yes: } 930-360 \mathrm{kN}(210,000- \\
\left.81,000 \mathrm{lb}_{\mathrm{f}}\right), \text { vacuum }\end{array}$ & \\
\hline \multirow[t]{2}{*}{ Restart capability } & \multirow[t]{2}{*}{$\begin{array}{l}\text { Yes, in } 3 \text { engines for } \\
\text { boostback, reentry, and landing }\end{array}$} & Yes, dual redundant TEA-TEB & \\
\hline & & pyrophoric igniters & \\
\hline Tank pressurization & Heated helium & Heated helium & \\
\hline Ascent attitude control: & \multirow[t]{2}{*}{ Gimbaled engines } & Gimbaled engine and & \\
\hline pitch, yaw & & nitrogen gas thrusters & \\
\hline $\begin{array}{l}\text { Ascent attitude } \\
\text { control: }\end{array}$ & Gimbaled engines & Nitrogen gas thrusters & \\
\hline $\begin{array}{l}\text { Coast/descent attitude } \\
\text { control }\end{array}$ & Nitrogen gas thrusters and grid fins & Nitrogen gas thrusters & Nitrogen gas thrusters \\
\hline Shutdown process & Commanded & Commanded & N/A \\
\hline Stage separation system & Pneumatic & $\mathrm{N} / \mathrm{A}$ & Pneumatic \\
\hline
\end{tabular}


The Falcon 9 fuel tank walls and domes are made from aluminum-lithium alloy. SpaceX rocket uses all-friction stir welded tank. The second stage tank of Falcon 9 is simply a shorter version of the first stage tank that uses the same tooling, material, and manufacturing techniques. This approach reduces manufacturing costs during vehicle production.[7]

All three cores of the Falcon Heavy in a structural calls Octaweb, which aimed at streamlining manufacture process,[16] and each core includes four extensible landing legs.[17] To control descent of boosters and center core through the atmosphere, SpaceX rocket uses small grid fins that deploy after separation.[18] Immediately after the side boosters separate, the center engine burns a few seconds in order to control the booster's trajectory safely away from the rocket.[17][19] The center core continues to fire until stage separation finish. The legs then deploy as the boosters landing softly on the ground after turn back to Earth. The landing legs are made of carbon fiber with aluminum honeycomb structure. The four legs stow along the sides of each core during liftoff and extend outward for landing.[20] Falcon Heavy specifications and characteristics are as follows:[21]. The Falcon Heavy uses a 4.5-meter (15 ft) [21] inter stage attached to the first stage core that is constructed of an aluminum honeycomb and surrounded by a carbon fiber face sheet plies. The overall length of vehicle at launch is 70 meters (230 $\mathrm{ft}$ ), and the total fueled mass is $1,420,000 \mathrm{~kg}$ $(3,130,000 \mathrm{lb})$. Without recovery of any stage, the Falcon Heavy can inject a 63,800 kg (140,700 lb) payload into a low Earth orbit, or $16,800 \mathrm{~kg}(37,000$ lb) to Venus or Mars [21]. The Falcon Heavy uses first-stage recovery systems to return the first stage booster at the launch site as well as return the first stage core by landing at an Autonomous Spaceport Drone Ship on the sea after primary mission completion. The recovery systems include four deployable landing legs that are locked on each firststage tank core during ascent. Excess propellant reserved for recovery operations of Falcon Heavy first-stage will be diverted in the primary thrusting stage so as to ensure sufficient performance margins of successful missions. The nominal payload capacity of launching to a geostationary transfer orbit (GTO) is $8,000 \mathrm{~kg}(18,000 \mathrm{lb})$. The Falcon Heavy can inject a $16,000 \mathrm{~kg}(35,000 \mathrm{lb})$ payload into GTO if only the two boosters are recovered.[21], with recovery of all three first-stage cores (the price per launch is $\$ 90$ million), 26,700 $\mathrm{kg}(58,900 \mathrm{lb})$ can be fully expendable ( $\$ 150$ million price per launch). The partially reusable Falcon Heavy falls into the heavy-lift range of launch systems, and is capable of lifting 20 to 50 metric tons into low Earth orbit under the classification system of NASA human spaceflight review panel.[22] A fully expendable Falcon Heavy is in the super heavy-lift category with a maximum payload of 64 tons launching to low Earth orbit. The payloads of initial envisioned concept model (Falcon 9-S9 2005) is 24,750 kg $(54,560 \mathrm{lb})$ launching to LEO, but in April 2011 this was projected to be up to $53,000 \mathrm{~kg}(117,000 \mathrm{lb})[23]$ and with GTO payloads up to $12,000 \mathrm{~kg}(26,000 \mathrm{lb})$.[24] Later reports of 2011 projected larger payloads are beyond LEO, including $19,000 \mathrm{~kg}(42,000 \mathrm{lb})$ to geostationary transfer orbit,[25] $16,000 \mathrm{~kg}(35,000$ lb) to trans lunar trajectory, and $14,000 \mathrm{~kg}(31,000$ 1b) of trans-Martian orbit to Mars.[26][27] 
Table 4

Capacity of Falcon Launch Vehicles

\begin{tabular}{|c|c|c|c|c|}
\hline \multicolumn{5}{|c|}{ Maximum theoretical payload capacity } \\
\hline \multirow[t]{3}{*}{ Destination } & \multicolumn{3}{|c|}{ Falcon Heavy } & Falcon 9 \\
\hline & \multirow{2}{*}{\begin{tabular}{|l|} 
Aug-13 \\
to Apr 2016 \\
\end{tabular}} & \multirow{2}{*}{$\begin{array}{l}\text { May-16 } \\
\text { to Mar } 2017\end{array}$} & \multirow[t]{2}{*}{ Since Apr 2017} & \\
\hline & & & & \\
\hline LEO $\left(28.5^{\circ}\right)$ expendable & $53,000 \mathrm{~kg}$ & $54,400 \mathrm{~kg}$ & $63,800 \mathrm{~kg}$ & $22,800 \mathrm{~kg}$ \\
\hline GTO $\left(27^{\circ}\right)$ expendable & $21,200 \mathrm{~kg}$ & $22,200 \mathrm{~kg}$ & $26,700 \mathrm{~kg}$ & $8,300 \mathrm{~kg}$ \\
\hline GTO $\left(27^{\circ}\right)$ reusable & $6,400 \mathrm{~kg}$ & $6,400 \mathrm{~kg}$ & $8,000 \mathrm{~kg}$ & $5,500 \mathrm{~kg}$ \\
\hline Mars & $13,200 \mathrm{~kg}$ & $13,600 \mathrm{~kg}$ & $16,800 \mathrm{~kg}$ & $4,020 \mathrm{~kg}$ \\
\hline Pluto & - & $2,900 \mathrm{~kg}$ & $3,500 \mathrm{~kg}$ & - \\
\hline
\end{tabular}

By late 2013, SpaceX raised the GTO payload of

Falcon Heavy up to $21,200 \mathrm{~kg}(46,700 \mathrm{lb})$.[28]. In

Figure 8: Diagram of Cost Model

April 2017, the projected LEO payload of Falcon

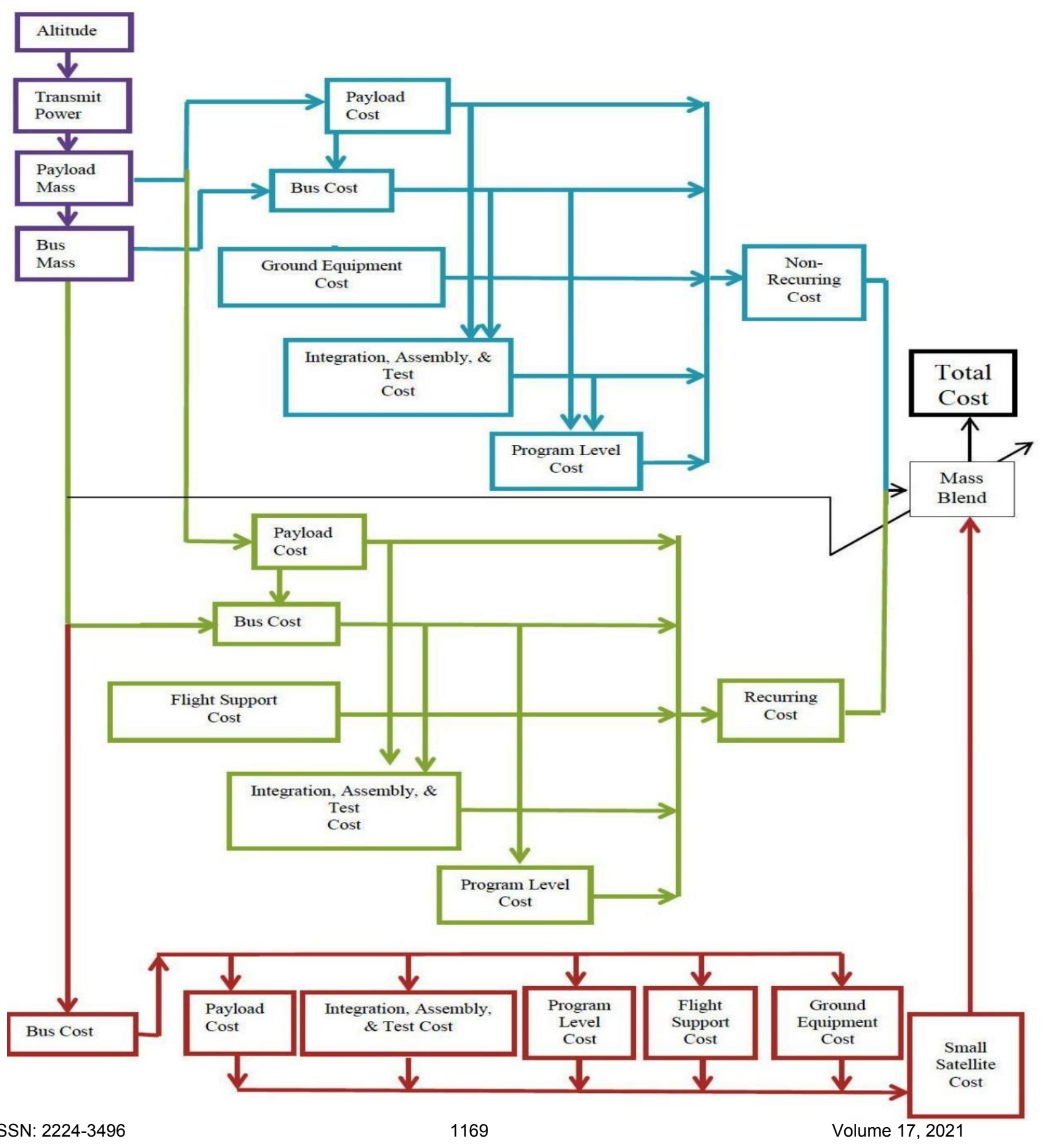


Table 5

SME-SMAD Cost Models

\begin{tabular}{|c|c|c|}
\hline \multicolumn{3}{|c|}{ SME-SMAD Cost Models, FY2010\$ } \\
\hline \multicolumn{3}{|c|}{ USCM8 Non-recurring Subsystem CERs in FY2010 Thousands of Dollars } \\
\hline Element & Equation & Variable \\
\hline 1 Spacecraft Bus & $110.2 * X$ & $\mathrm{X}=$ Spacecraft weight $(\mathrm{kg})$ \\
\hline 2 Payload & $618 * X$ & $\begin{array}{c}\mathrm{X}=\text { Communications subsystem } \\
\text { weight }(\mathrm{kg})\end{array}$ \\
\hline $\begin{array}{c}3 \text { Integration, Assembly, } \\
\text { and Test }\end{array}$ & $0.195 * \mathrm{X}$ & $\begin{array}{l}\mathrm{X}=\text { Spacecraft bus + Payload non- } \\
\text { recurring cost }(\$ \mathrm{~K})\end{array}$ \\
\hline 4 Program Level & $0.414 * \mathrm{X}$ & $\begin{array}{c}\mathrm{X}=\text { Space vehicle and IA\&T non- } \\
\text { recurring cost }(\$ K)\end{array}$ \\
\hline $\begin{array}{l}5 \text { Aerospace Ground } \\
\text { Equipment (AGE) }\end{array}$ & $0.421 * X 10.907 * 2.244 \times 2$ & $\begin{array}{c}\mathrm{X} 1=\text { Spacecraft bus non-recurring } \\
\text { cost }(\$ \mathrm{~K}) \\
\mathrm{X} 2=0 \text { for comm sats } \\
\mathrm{X} 2=1 \text { for non-comm sats }\end{array}$ \\
\hline \multicolumn{3}{|c|}{ USCM8 Spacecraft Bus Recurring T1 CERs in FY2010 Thousands of Dollars } \\
\hline Element & Equation & Variable \\
\hline 1 Spacecraft Bus & $289.5 * \mathrm{X}_{0.716}$ & $\mathrm{X}=$ Spacecraft weight $(\mathrm{kg})$ \\
\hline 2 Payload & $189 * X$ & $\begin{array}{c}\mathrm{X}=\text { Communications payload } \\
\text { weight }(\mathrm{kg})\end{array}$ \\
\hline $\begin{array}{c}3 \text { Integration, Assembly, } \\
\text { and Test }\end{array}$ & $0.124 * X$ & $\begin{aligned} \mathrm{X}= & \text { Spacecraft Bus }+ \text { Payload } \\
& \text { Recurring Cost }(\$ K)\end{aligned}$ \\
\hline 4 Program Level & $0.320 * \mathrm{X}$ & $\begin{array}{c}\mathrm{X}=\text { Spacecraft Recurring Cost } \\
(\$ \mathrm{~K})\end{array}$ \\
\hline 5 Flight Support & 5850 & - \\
\hline \multicolumn{3}{|c|}{ SSCM Total Non-recurring Cost (development plus one protoflight unit) } \\
\hline Element & Equation & Variable \\
\hline 1 Spacecraft Bus & $1064+35.5 * \mathrm{X}_{1.261}$ & $\mathrm{X}=$ Spacecraft weight $(\mathrm{kg})$ \\
\hline 2 Payload & $0.4 * \mathrm{X}$ & $\begin{array}{c}\mathrm{X}=\text { Spacecraft Bus Total Cost } \\
(\$ \mathrm{~K})\end{array}$ \\
\hline $\begin{array}{c}3 \text { Integration, Assembly, } \\
\text { and Test }\end{array}$ & $0.139 * X$ & $\mathrm{X}=$ Spacecraft Bus Total Cost $(\$ K)$ \\
\hline 4 Program Level & $0.229 * \mathrm{X}$ & $\mathrm{X}=$ Spacecraft Bus Total Cost $(\$ K)$ \\
\hline 5 Flight Support & $0.061 * X$ & $\mathrm{X}=$ Spacecraft Bus Total Cost $(\$ K)$ \\
\hline $\begin{array}{l}6 \text { Aerospace Ground } \\
\text { Equipment (AGE) }\end{array}$ & $0.066^{*} \mathrm{X}$ & $\mathrm{X}=$ Spacecraft Bus Total Cost $(\$ K)$ \\
\hline
\end{tabular}


Heavy was raised from $54,400 \mathrm{~kg}(119,900 \mathrm{lb})$ to $63,800 \mathrm{~kg}(140,700 \mathrm{lb})$. The maximum payload is achieved when the rocket flies a fully expendable launch profile, without recovering any of three firststage boosters.[31]

With the expendable core booster and recoverable two side-boosters, it is estimated that the payload penalty could be around $10 \%$, and still yield over 57 metric tons of lift capability to LEO.[29] Returning all three boosters to the launch site rather than landing on drone ships would yield about 30 metric tons of payload to LEO.[30]

\section{SME-SMAD Cost Models.}

This paper explains the specific equations used for the spacecraft cost. Using Equation (1), the cost function calculates cost in 2010 thousands of dollars for the entire system. The three cost models included in the cost function are detailed in Table 5 [1]. Figure 8 is Diagram of three cost modules developed by SME-SMAD and shows how they are combined as a system cost model.

Equation (6) represents the USCM8 NRE model in Table 5 .

$$
\begin{gathered}
\text { Cost }_{\text {NRE }}=\text { Cost }_{s / c \_b u s}+\text { Cost }_{\text {Payload }}+ \\
\text { Cost }_{\text {IA\&T }}+\text { Cost }_{\text {program_level }}+\text { Cost }_{\text {AGE }}
\end{gathered}
$$

where,

$$
\begin{aligned}
& \text { Cost }_{S / \text { c bus }_{-}}=\text {cost of the spacecraft bus } \\
& \text { Cost }_{\text {Payload }}=\text { cost of the payload } \\
& \text { Cost }_{\text {IA\&T }} \\
& =\text { cost of integration, assembly, and test }
\end{aligned}
$$

Cost $_{\text {program_level }}=$ cost of the program level
$\operatorname{Cost}_{A G E}$

$=$ cost of the aerospace ground equipment

Each component seen above is defined respectively in Table 5.

Equation 7 illustrates the USCM8 recurring cost in Table 5.

$$
\begin{aligned}
& \operatorname{Cost}_{\text {recurring }}=\text { Cost }_{s / c_{-} \text {bus }}+\text { Cost }_{\text {Payload }}+ \\
& \operatorname{Cost}_{\text {IA\&T }}+\text { Cost }_{\text {program_level }}+\text { Cost }_{\text {flight_support }}
\end{aligned}
$$

where, the same notation is used from above. Equation (8) represents the SSCM NRE cost in Table 5.

$$
\begin{aligned}
& \operatorname{Cost}_{S S C M}=\text { Cost }_{S / c_{-} \text {bus }}+\text { Cost }_{\text {Payload }}+ \\
& \operatorname{Cost}_{\text {IA\&T }}+\text { Cost }_{\text {program_level }}+ \\
& \operatorname{Cost}_{\text {flight_support }}+\text { Cost }_{A G E}
\end{aligned}
$$

Where, each component is defined respectively in Table 5.

Let's take current GPS constellation as a realistic example to compare the cost model's results. The cost value is compared with the cost of GPS Block III satellites. To compare the cost produced by the algorithm with the price per unit of GPS Block III satellites, the launch cost was subtracted from the total cost on the basis of MOGA. The total GPS cost is $\$ 4.21$ billion and according to the unit cost of GPS Block III [32], the cost on the basis of MOGA is within $22 \%$ of total launch cost computed by other methods value.

\section{Cost Function for Availability}

There are three test cases conducted within the LEO altitude simulations. Each test case results in Pareto 
fronts that demonstrate a higher cost for improvement on performance. The LEO test cases get better performance as altitude increased. The LEO constellation optimized by MOGA is expected on tradeoffs between PDOP and cost, and the results are also dominated in the MEO constellation.

Figure 9 illustrates the three LEO Pareto front's comparison. The design solutions for different altitude constellations are compared by the PDOP and cost values. The most desirable solution is around the concave portion of the Pareto front. These solutions are chosen from the individual Pareto fronts rather than values in Figure 9 since the scaling for result comparison makes it difficult to display the concave portion. See Appendix C for the Pareto fronts of the individual test cases.

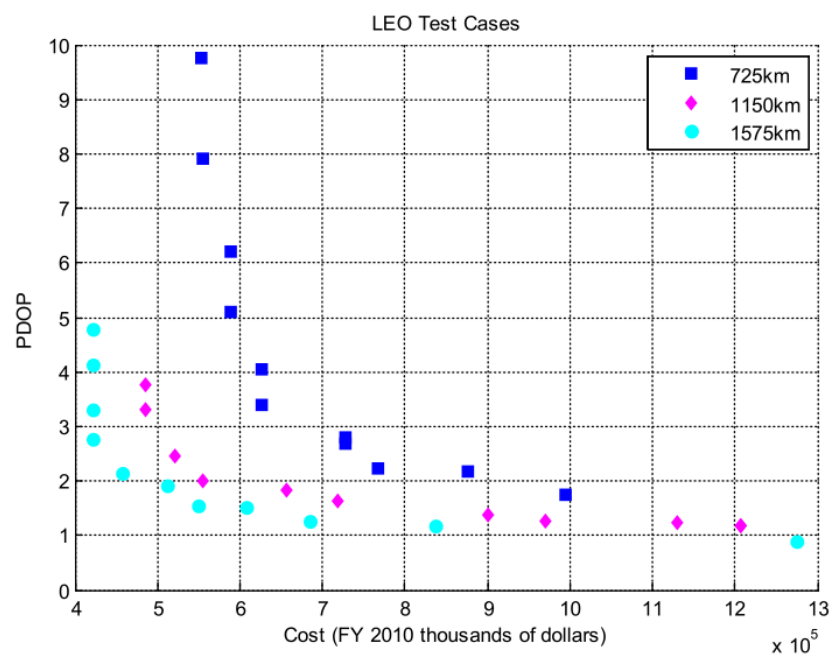

Figure 9: PDOP and cost of LEO Pareto fronts

The lowest altitude constellation resulted in lower PDOP (Position solution Dilution of Precision) values and higher cost. This trend continued as the middle altitude constellation has higher PDOP and cost values than the highest altitude. The points selected as the desirable solutions show that the highest altitude constellation completely dominated the Pareto fronts at lower altitudes. Table 6 illustrates that when PDOP remains close to two, the cost decreases dramatically as altitude increases. Table 7 demonstrates that when cost remains relatively the same, PDOP decreases as altitude increases, but not as dramatically as the cost. Therefore, as altitude increases, there is more benefit in the cost than in the performance of the constellation designs produced within the LEO altitude range.

Table 6: Cost Tradeoff for LEO Test Cases

\begin{tabular}{lrr}
\hline & PDOP & Cost (\$B) \\
\hline $725 \mathbf{~ k m}$ & 2 & 0.99 \\
$1150 \mathbf{~ k m}$ & 2 & 0.55 \\
$1575 \mathbf{~ k m}$ & 2 & 0.45 \\
\hline
\end{tabular}

Table 7: PDOP Tradeoff for LEO Test Cases

\begin{tabular}{lrc}
\hline & PDOP & Cost (\$B) \\
\hline $\mathbf{7 2 5} \mathbf{~ k m}$ & 3.34 & 0.6 \\
$\mathbf{1 1 5 0} \mathbf{~ k m}$ & 1.82 & 0.6 \\
$\mathbf{1 5 7 5} \mathbf{~ k m}$ & 1.51 & 0.6 \\
\hline
\end{tabular}

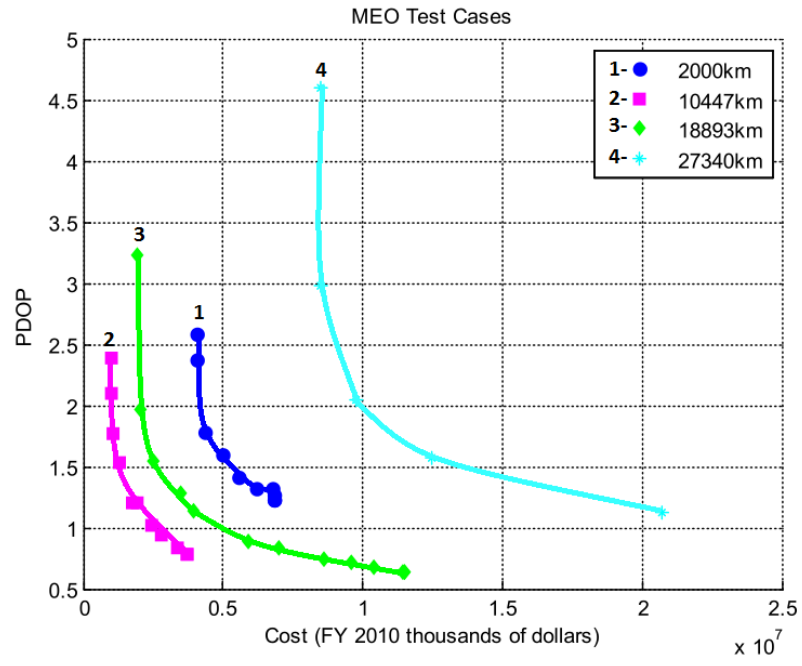

Figure 10: PDOP and cost of MEO Pareto fronts

Within the MEO altitude range, there are four test cases. Each test case illustrates an increase in cost for better PDOP values. The MEO simulation 
produces lower PDOP values than those in the LEO constellations. The results verify the consistent trend of PDOP and cost. To determine the difference of results in the MEO test cases, the four Pareto fronts are compared in Figure 10. The number of MEO constellation satellites required for the PDOP values decreases as the altitude increases. Unlike the LEO test cases, there was a large difference in cost for MEO test cases when PDOP remained constant. The cost trend of MEO constellation firstly drops down then turns up along with the altitude raises. It can be seen in Table 8 that PDOP remains constant around 1.5 , the first case or altitude constellation results in a higher cost than two middle altitude constellations. The last three altitude constellations show an increase in cost along with altitude increases. The variations in cost as altitude increases are larger than in the LEO test cases.

Table 8: Cost Tradeoff for MEO Test Cases

\begin{tabular}{lcc}
\hline & PDOP & Cost (\$B) \\
\hline $\mathbf{2 0 0 0} \mathbf{~ k m}$ & 1.5 & 4.9 \\
$\mathbf{1 0 4 4 7} \mathbf{~ k m}$ & 1.5 & 1.3 \\
$\mathbf{1 8 8 9 3} \mathbf{~ k m}$ & 1.5 & 2.5 \\
$\mathbf{2 7 3 4 0} \mathbf{~ k m}$ & 1.5 & 12.4 \\
\hline
\end{tabular}

Table 9: PDOP Tradeoff for MEO Test Cases

\begin{tabular}{lcc}
\hline & PDOP & Cost (\$B) \\
\hline $\mathbf{2 0 0 0} \mathbf{~ k m}$ & 2.37 & 3.9 \\
\hline
\end{tabular}

\begin{tabular}{lcc}
\hline $\mathbf{1 0 4 4 7} \mathbf{~ k m}$ & 0.78 & 3.9 \\
$\mathbf{1 8 8 9 3} \mathbf{~ k m}$ & 1.14 & 3.9 \\
$\mathbf{2 7 3 4 0} \mathbf{~ k m}$ & None & 3.9 \\
\hline
\end{tabular}

When the cost remains at $\$ 3.9$ billion dollars, Table 9 illustrates that the second MEO altitude constellation possesses the lowest PDOP value. The fourth case or altitude constellation does not have a PDOP value related to the cost because it is too much for this altitude. The changes in PDOP as altitude increases are similar to the results of the LEO constellation. To explain the unique trend of the system cost in MEO range, spacecraft cost and launch cost of the design solution for each test case are determined. The solutions that PDOP value equals 1.5 are selected from each altitude case for the comparison of cost. Due to the larger difference in altitude used for the MEO range, there is a larger difference in the number of satellites for each test case. To determine whether the cost is quantity driven or size driven, Table 10 illustrates the different cost values against the same performance in four test cases of the MEO constellations.

The fourth test case or altitude constellation, results in the most highest cost as seen in Table 9. This is because large size satellites are required for the orbit altitude. With comparing the other test cases, the

Table 10

Cost Comparisons of MEO Test cases

\begin{tabular}{|c|c|c|c|c|c|c|c|c|}
\hline $\begin{array}{c}\text { MEO } \\
\text { Test } \\
\text { Case }\end{array}$ & $\begin{array}{c}\text { Altitude } \\
(\mathrm{km})\end{array}$ & PDOP & \#Planes & \# Satellites & $\begin{array}{c}\text { \# Launch } \\
\text { Vehicles }\end{array}$ & $\begin{array}{c}\text { Cost Per } \\
\text { Satellite } \\
(\$ B)\end{array}$ & $\begin{array}{c}\text { Total } \\
\text { Satellite } \\
\text { Cost }(\$ B)\end{array}$ & $\begin{array}{c}\text { Total } \\
\text { Launch } \\
\text { Cost }(\$ B)\end{array}$ \\
\hline 1 & 2000 & 1.5 & 8 & 88 & 8 & 0.42 & 36.80 & 0.45 \\
\hline 2 & 10447 & 1.5 & 4 & 32 & 4 & 0.85 & 27.30 & 0.23 \\
\hline 3 & 18893 & 1.5 & 5 & 25 & 5 & 1.92 & 48.00 & 0.28 \\
\hline 4 & 27340 & 1.5 & 4 & 24 & 20 & 11.30 & 271.00 & 1.14 \\
\hline
\end{tabular}


first test case or the lowest altitude MEO constellation also possessed the expensive results, thus the spacecraft cost for this altitude owns the primary prices for the high cost reason. Table 10 shows that the price for this test case is driven by the large amount of satellites as 88. This altitude requires large number of satellites to produce PDOP value less than six; consequently it requires more number of launch vehicles. The third test case produces more expensive results than the second test case, this is driven by the satellite size. After comparing the MEO cost results, the second test case possesses the lowest cost when PDOP (Position solution Dilution of Precision) is set at 1.5 and the least PDOP value when cost remained constant. The last test case of altitude is HEO simulation. An altitude range of $35786-35796 \mathrm{~km}$ is used for this test case. There are fewer design solutions that can make PDOP values less than six. Therefore, very few Pareto fronts are obtained. The solutions continue to follow the expected trend of an inverse relationship between PDOP and cost.

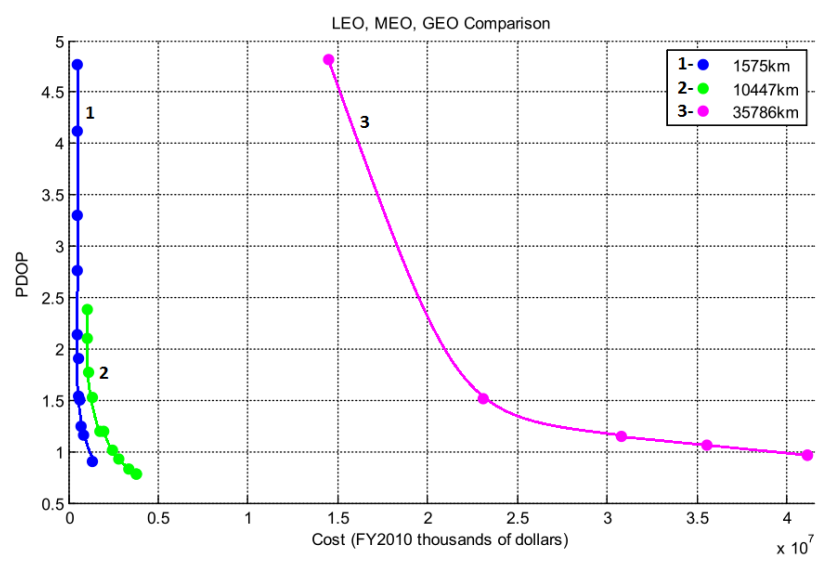

Figure 11: PDOP and cost of Pareto fronts in LEO, MEO, and GEO constellations

Since there is so much difference in cost among LEO, MEO, and HEO constellations, only dominating Pareto fronts of LEO and MEO simulations are used to compare with the GEO designs. Figure 11 shows the comparison of the Pareto fronts for different altitudes. The cost of the designs increases dramatically from LEO to $\mathrm{HEO}$ altitude when PDOP is held by constant value 1.5 (Table 11). The LEO design resulted in a lower cost than the MEO and GEO designs when PDOP equals 1.5. At the same cost of $\$ 1.3$ billion, the LEO design produces a lower PDOP value than MEO results that are listed in Table 12. The price comparison is only available for LEO and MEO cases. There are no common performance between GEO and the lower altitudes at this cost.

Table 11: Cost Tradeoff for LEO, MEO, and HEO constellations

\begin{tabular}{lcc}
\hline & PDOP & Cost (\$B) \\
\hline LEO & 1.5 & 0.61 \\
MEO & 1.5 & 1.31 \\
GEO & 1.5 & 23.10 \\
\hline
\end{tabular}

Table 12: PDOP Tradeoff for LEO, MEO, and GEO constellations

\begin{tabular}{lcc}
\hline & PDOP & Cost (\$B) \\
\hline LEO & 0.90 & 1.3 \\
MEO & 1.53 & 1.3 \\
GEO & None & 1.3 \\
\hline
\end{tabular}

To compare the cost of designs at different altitude ranges, Figure 12 illustrates cost against altitude increases, and Figure 13 shows cost against the number of satellites increases. Figure 12 demonstrates that the GEO constellation designs are more expensive than the MEO and LEO designs. 


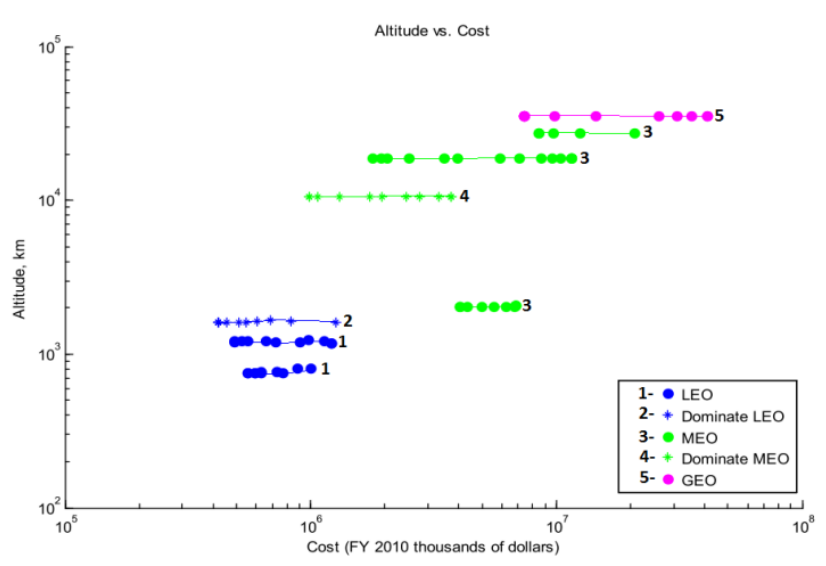

Figure 12: Altitude vs. Cost Comparison for LEO, MEO and HEO constellations

Some of the MEO designs are more expensive than the LEO designs but some are not. There are some solutions that overlap with the LEO results. The number of LEO satellites is greater than HEO for the same propose but some LEO constellations possess the same number of satellites as the MEO constellations. For each altitude constellation, the number of satellites increases along with the cost increases. The trend of altitude versus cost is shown in Figure 13.

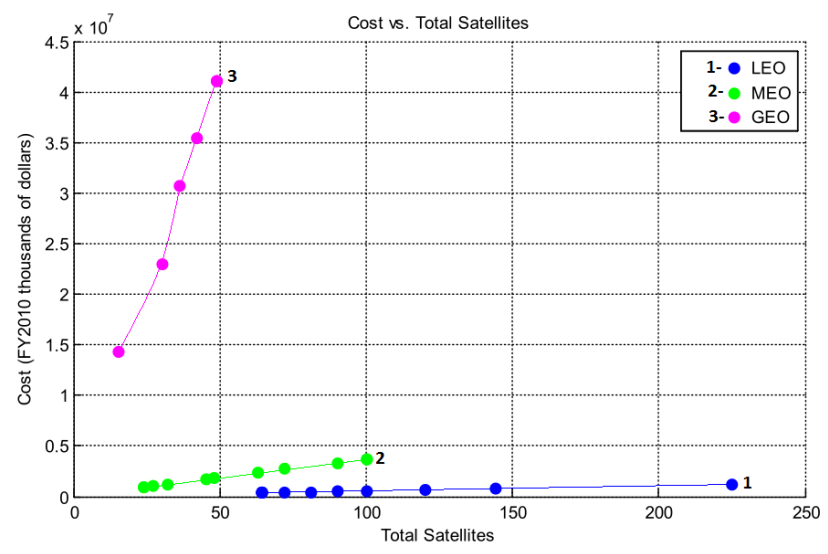

Figure 13: Cost vs. Total Satellites for LEO, MEO and HEO constellations

PDOP (Position solution Dilution of Precision) is used for evaluating performance of different altitude constellations respecting to number of satellites.

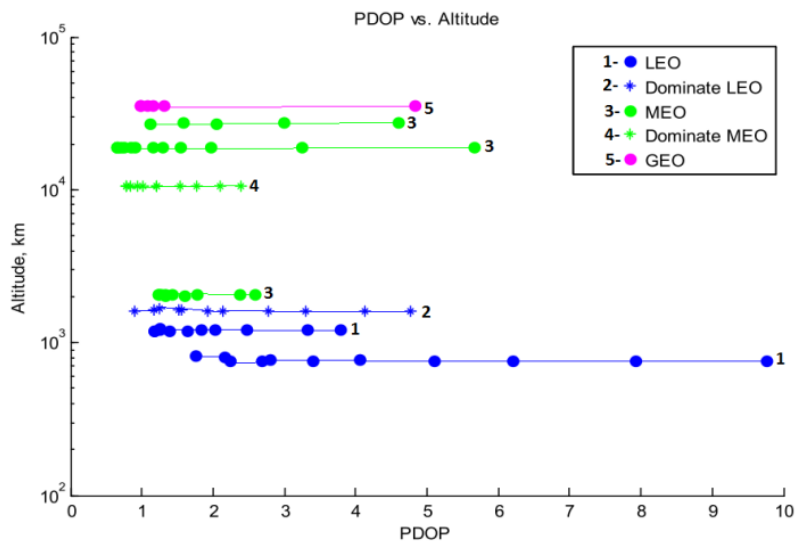

Figure 14: PDOP vs. Altitude for LEO, MEO and HEO constellations

Figure 14 illustrates PDOP with respect to altitude, and it shows that each altitude constellation may have different the PDOP values. All altitude constellations can produce similar PDOP values around two. Figure 15 shows the relations between PDOP values and the number of satellites in each altitude range of constellation. The LEO constellations generate higher PDOP values than MEO and HEO. This figure also illustrates that the constellations in three altitude ranges may produce similar PDOP values, but LEO constellation requires more satellites to reach those values.

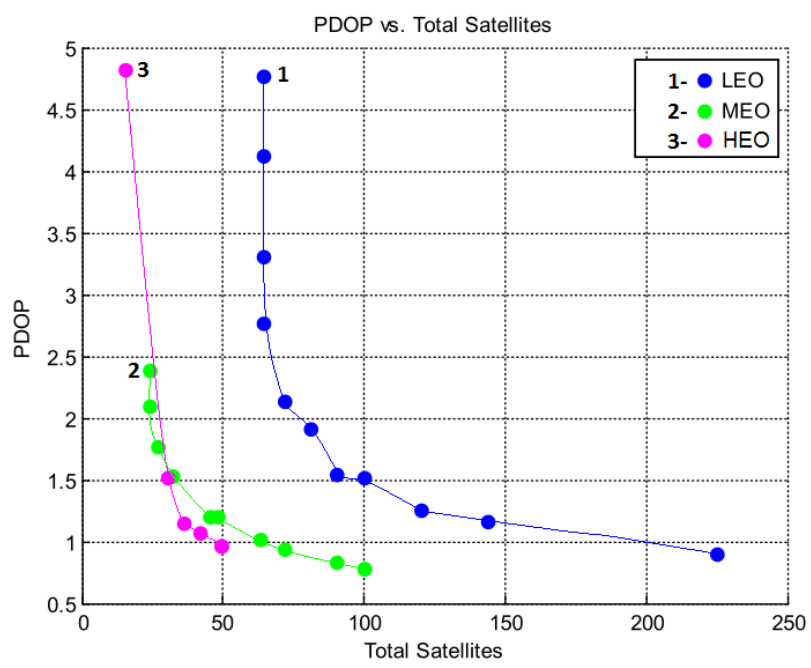

Figure 15: PDOP vs. Total Satellites for LEO, MEO and HEO constellations 
The design tool introduced in this research has the ability to analyze multiple satellite types. Therefore, three hybrid constellations are analyzed by LEOMEO hybrid, LEO-HEO hybrid, and MEO-HEO hybrid. Figure 16 demonstrates the Pareto front of the LEO-MEO hybrid designs and individual LEO or MEO altitude designs. The Pareto front of hybrid constellation falls very close to the MEO Pareto front. For a given PDOP value, the LEO results still possess lower cost than MEO and LEO-MEO hybrid constellations. The hybrid cost value is much similar to the MEO value but not to the LEO (as Table 13). When setting the lower and upper bounds of design parameters for the test cases, both MEO and hybrid altitudes possess a lower bound that MOGA value equals two for determining number of planes and number of satellites per plane. MOGA do not have the option of setting only for MEO or LEO satellite constellations.

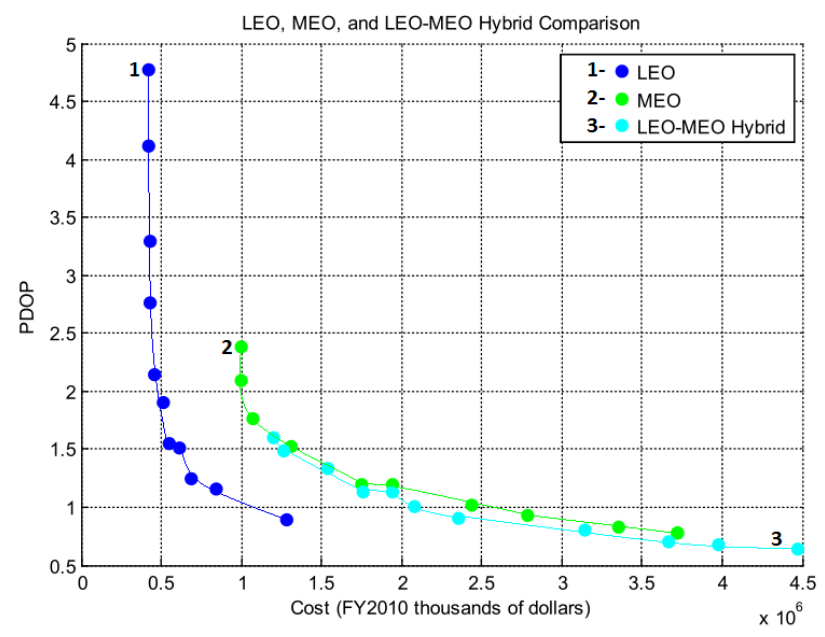

Figure 16: Comparison of LEO, MEO, and LEO-MEO Hybrid constellations

The cost of hybrid design is close to the individual MEO cost and MOGA value could be same in the LEO constellation design, but MEO constellations require at least four satellites. This is a possible reason that the cost of hybrid design is greater than the individual LEO designs.

Table 13: Cost Tradeoff for LEO, MEO, and LEO-MEO Hybrid

\begin{tabular}{lcc}
\hline & PDOP & Cost (\$B) \\
\hline LEO & 1 & 1.27 \\
MEO & 1 & 2.08 \\
LEO-MEO Hybrid & 1 & 2.44 \\
\hline
\end{tabular}

For a required cost of $\$ 1.3$ billion, the PDOP values show an increase from LEO to MEO and hybrid, LEO constellation possesses the lowest value and the hybrid constellation possess the highest value. As demonstrated in Table 14, the PDOP value of the hybrid design is much closer to the MEO value. It means that there is no additional benefit of utilizing LEO-MEO hybrid constellation.

Table 14: PDOP Tradeoff for LEO, MEO, and LEO-MEO Hybrid

\begin{tabular}{lcc}
\hline & PDOP & Cost (\$B) \\
\hline LEO & 0.90 & 1.3 \\
MEO & 1.49 & 1.3 \\
LEO-MEO Hybrid & 1.53 & 1.3 \\
\hline
\end{tabular}

Figure 17 compares the trends of PDOP and total number of satellites in each altitude range along with the LEO-MEO hybrid constellation designs. The hybrid design falls right in the LEO and MEO designs. The hybrid PDOP values are lower than LEO, but it requires the same number of satellites as MEO when PDOP is greater than one and the same number of satellites as LEO for PDOP less than one. 


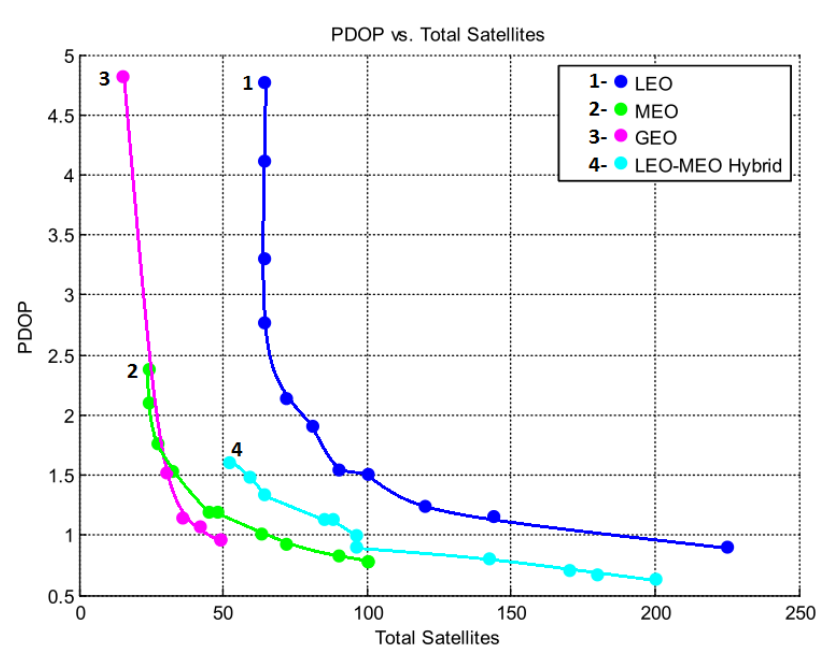

Figure 17: PDOP vs. Total Satellites for LEO-MEO Hybrid constellations

Table 15 illustrates the separate number of LEO and MEO satellites within the LEO-MEO hybrid designs. Along whit PDOP increases, the number of required satellites decreases. If one type of constellation is applied, only the same numbers of LEO satellites are required or using fewer MEO satellites. This could be a result of the LEO satellites costing less than the MEO due to their smaller size.

Table 15: Number of LEO and MEO Satellites in the Hybrid Designs

\begin{tabular}{lcc}
\hline PDOP & $\begin{array}{c}\text { Total LEO } \\
\text { Satellites }\end{array}$ & $\begin{array}{c}\text { Total MEO } \\
\text { Satellites }\end{array}$ \\
\hline $\mathbf{0 . 6 4}$ & 100 & 100 \\
$\mathbf{0 . 6 7}$ & 90 & 90 \\
$\mathbf{0 . 7 1}$ & 90 & 80 \\
$\mathbf{0 . 8 0}$ & 72 & 70 \\
$\mathbf{0 . 9 0}$ & 42 & 54 \\
$\mathbf{1 . 0 0}$ & 56 & 40 \\
$\mathbf{1 . 1 3}$ & 45 & 40 \\
$\mathbf{1 . 1 3}$ & 56 & 32 \\
$\mathbf{1 . 3 3}$ & 32 & 32 \\
$\mathbf{1 . 4 8}$ & 35 & 24 \\
$\mathbf{1 . 6 0}$ & 28 & 24 \\
\hline
\end{tabular}

Figure 18 illustrates the individual LEO and HEO Pareto fronts compared to the LEO-HEO hybrid Pareto front. The hybrid front falls close to the HEO
Pareto front, and showed some overlap in cost. Table 16 and Table 17 show the cost and PDOP tradeoffs.

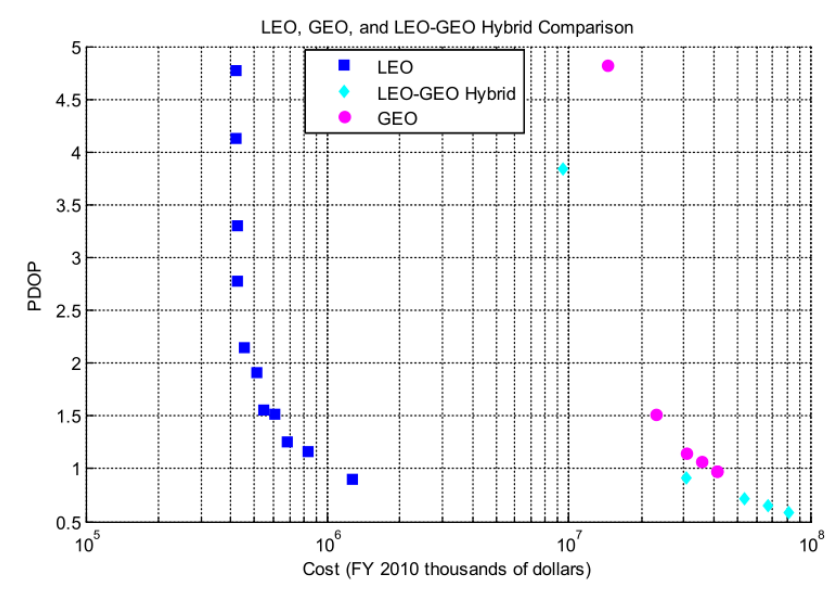

Figure 18: PODP vs cost of LEO, GEO, and LEO-GEO Hybrid Constellations

When PDOP values take one, the individual LEO design has the lowest cost as 1.27 billion USD, and the individual HEO design has the highest cost as 41.13 billion USD (Table 16). The LEO-HEO hybrid cost is close to the cost value of HEO. This is attributed to the same issue mentioned in the LEOMEO hybrid. Figure 18 illustrates the number of satellites at the HEO altitude and LEO altitude within the hybrid designs. The number of HEO satellites is never larger than the number of LEO satellites.

Table 16: Cost Tradeoff for LEO, GEO, and LEO-HEO Hybrid

\begin{tabular}{lrc}
\hline & PDOP & Cost (\$B) \\
\hline LEO & 1 & 1.27 \\
GEO & 1 & 41.13 \\
LEO-GEO Hybrid & 1 & 30.69 \\
\hline
\end{tabular}


Table 17: PDOP Tradeoff for LEO, GEO, and LEO-GEO Hybrid

\begin{tabular}{lcc}
\hline & PDOP & Cost (\$B) \\
\hline LEO & None & 30 \\
GEO & 1.16 & 30 \\
LEO-GEO Hybrid & 0.91 & 30 \\
\hline
\end{tabular}

The MOGA is applied to select satellites for both altitudes, so the hybrid constellation cost would automatically be driven to higher cost than LEO. In Table 17, when PDOP is analyzed for a constant cost as $\$ 30$ billion, the LEO altitude does not have a design result because it has a much lower cost value. The LEO-MEO hybrid produces in a slightly lower PDOP value than HEO. The cost and PDOP values of the hybrid designs remain closer to the higher individual altitude just as was shown in Figure 19. The LEO-MEO hybrid result shows the change in the number of satellites for each altitude constellation along with the PDOP decreases. The hybrid design shows a similar number of satellites required by LEO when the PDOP is equal to one. The MEO-HEO hybrid constellation is compared to two individuals of MEO and HEO designs in Figure 20. The hybrid constellation's Pareto front falls in between the MEO and HEO Pareto fronts.

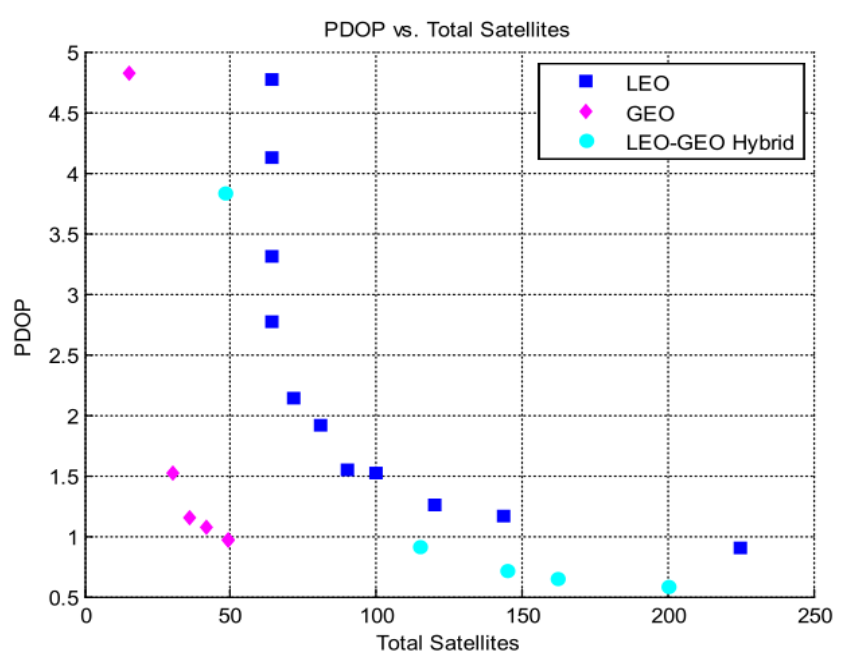

Figure 19: PDOP vs. Total Satellites for LEO, GEO, and LEO-HEO Hybrid constellations

Table 18: Cost Tradeoff for MEO, HEO, and MEO-HEO Hybrid constellations

\begin{tabular}{lcc}
\hline PDOP & $\begin{array}{c}\text { Total LEO } \\
\text { Satellites }\end{array}$ & $\begin{array}{c}\text { Total GEO } \\
\text { Satellites }\end{array}$ \\
\hline $\mathbf{0 . 5 8}$ & 100 & 100 \\
$\mathbf{0 . 6 5}$ & 81 & 81 \\
$\mathbf{0 . 7 1}$ & 81 & 64 \\
$\mathbf{0 . 9 0}$ & 80 & 35 \\
$\mathbf{3 . 8 3}$ & 40 & 8 \\
\hline
\end{tabular}

Table 19: PDOP Tradeoff for MEO, HEO, and MEO-HEO Hybrid constellations

\begin{tabular}{lcc}
\hline & PDOP & Cost (\$B) \\
\hline MEO & 1 & 2.44 \\
GEO & 1 & 41.13 \\
MEO-GEO Hybrid & 1 & 18.10 \\
\hline
\end{tabular}

Table 18 and Table 19 demonstrate cost and PDOP tradeoffs. When PDOP value takes one, the MEO results possess the lowest cost, and HEO results are the highest cost. The difference between hybrid MEO-HEO cost and HEO cost is larger than discussed in the other hybrid constellation results. The MEO-HEO cost is similarly higher than the 
MEO results for the same reason in the other hybrid cases. For a cost of $\$ 30$ billion, there is not a MEO design available because the MEO designs possess a much less cost value. The MEO-HEO hybrid produces less PDOP value than the HEO results but they are relatively close each other.

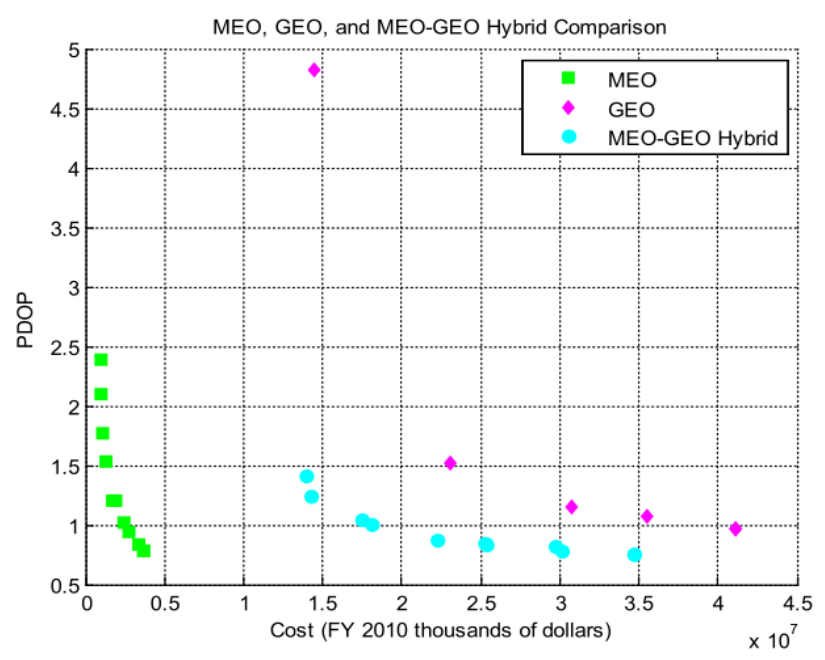

Figure 20: PDOP vs cost of MEO, GEO, and MEO-GEO

Hybrid Constellations

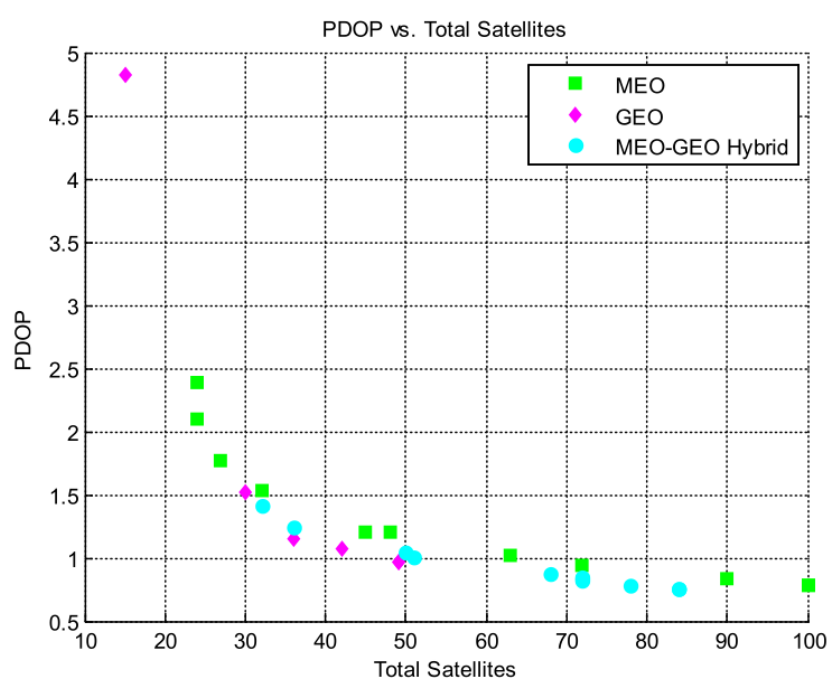

Figure 21: PDOP vs. Total Satellites for MEO-HEO Hybrid constellation

The relations between PDOP values and number of required satellites in MEO, HEO and MEO-HEO hybrid constellations are displayed in Figure 21. For PDOP value near one, MEO constellation needs more satellites than the HEO and MEO-HEO constellations need that both require the same number of satellites. The same trend continues that there are fewer satellites required for high altitude constellation than the hybrid one.

\section{PDOP vs. Constellation Parameters}

The results from each of the test cases demonstrate similar trends that the number of planes and satellites per plane change along with PDOP (Position solution Dilution of Precision) decrease. The inclination of constellation remains almost unchanged for most of the altitudes. The following graphs are directed against LEO, MEO and HEO trends. The MEO and HEO results can be combined since they are relatively similar. Figure 22 illustrates the relationship between PDOP and the number of orbit planes for different altitude LEO constellations. As expected, the PDOP values decrease when the number of orbit planes increases. For the same number of orbit planes, there are multiple PDOP points on this account that some solutions possessing more satellites per plane. For PDOP value above two, the number of planes decreases as the altitude increases. 


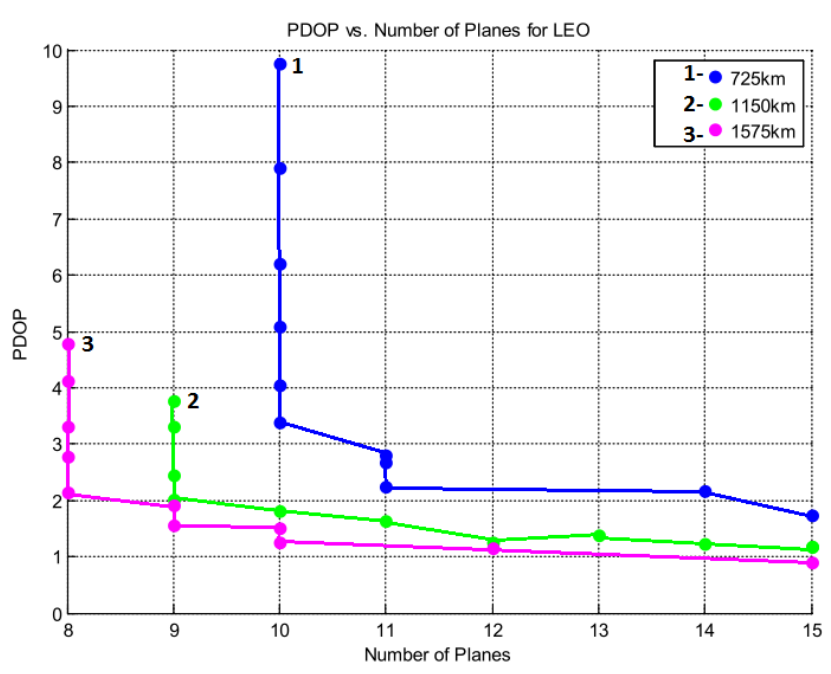

Figure 22: PDOP vs. Number of Planes for LEO constellation

In Figure 23 PDOP value decreases when the number of satellites per orbit plane increases for different altitude constellations. This is the same trend seen as the number of orbit planes due to the total number of satellites has impact on PDOP value. For the same number of satellites per plane, these points show that show a decrease of PDOP as the altitude increases. This is attribute to the points possessing more orbital planes. Some solutions possess the same number of orbit planes and satellites per plane, but still demonstrate different PDOP values. This will be analyzed later with the effects of inclination.

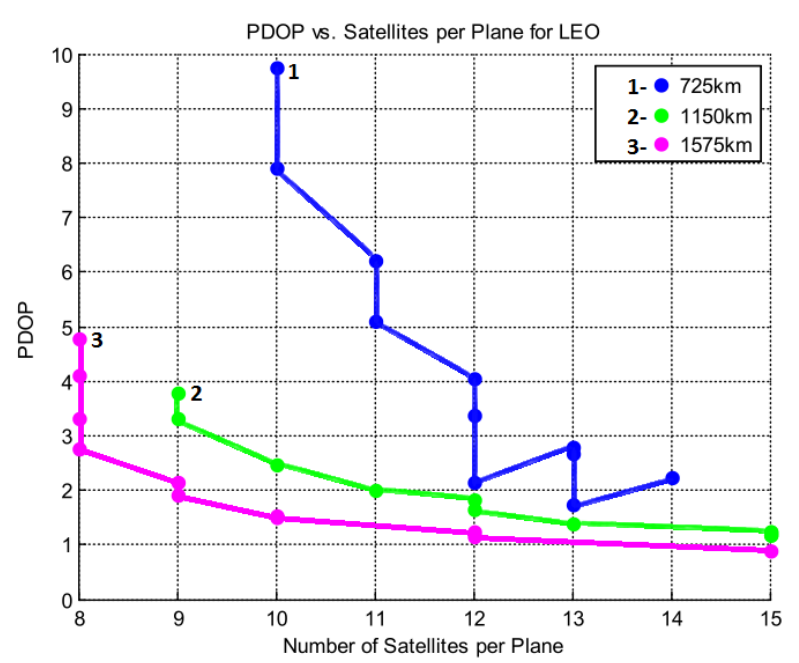

Figure 23: PDOP vs. number of Satellites per Plane for LEO

Figure 24 demonstrates the relationship between the number of satellites per plane and the number of orbit planes. The difference between the number of orbit planes and the number of satellites per plane is always less than three. For the upper lever altitude constellation, the trend of relation between satellites per plane and orbit planes appears a staircase effect. As the number of orbit planes increases with altitude level goes up, the number of satellites per plane either remains the same or increases as well.

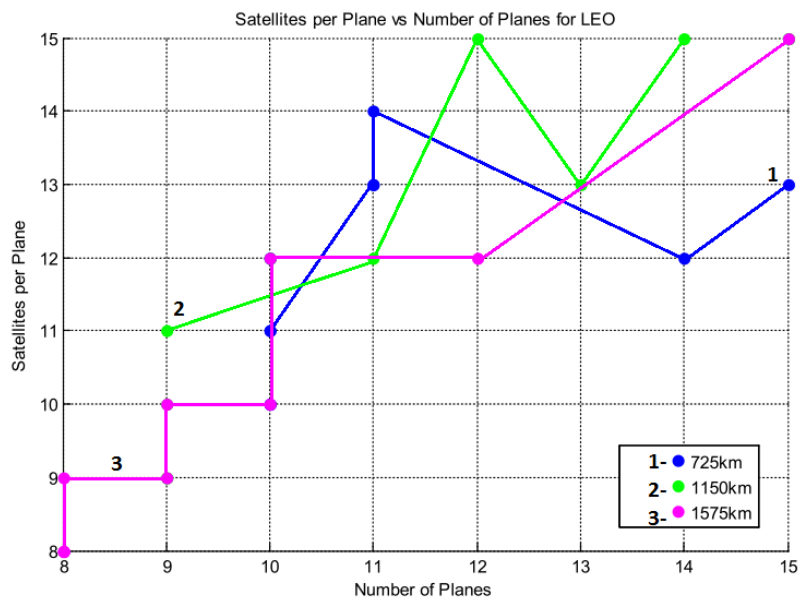

Figure 24: Satellites per Plane vs. Number of Orbit Planes for LEO

The third test case that results in dominating Pareto front in LEO constellations, illustrates a roughly 
linear trend of relation between the number of satellite per plane and the number of planes. Equation (9) represents the trend.

spp $=0.9757 *$ planes +0.5951

where $\mathrm{R}^{2}=0.91$. The inclination results are not demonstrated as the same trend as seen with the previous two parameters. The inclination do not decrease or increase against PDOP values of the lower level altitude constellations, but for the upper level altitude constellations, the inclination showed an increase along with increase of PDOP value. This is the only case when inclination shows this specific trend. For all the LEO constellations, the inclination remains within a 10 degree range. In Figure 25, For $725 \mathrm{~km}$ altitude, the inclination takes 54 to 58 degrees. The results of altitude at $1150 \mathrm{~km}$ take 48 to 58 degrees, and lastly, for $1575 \mathrm{~km}$ altitude, the inclination remains between 36 and 46 degrees. Suppose PDOP value equals two, the inclination is roughly equal for the lower level altitude constellations, but the upper level altitude constellations have the lowest inclination.

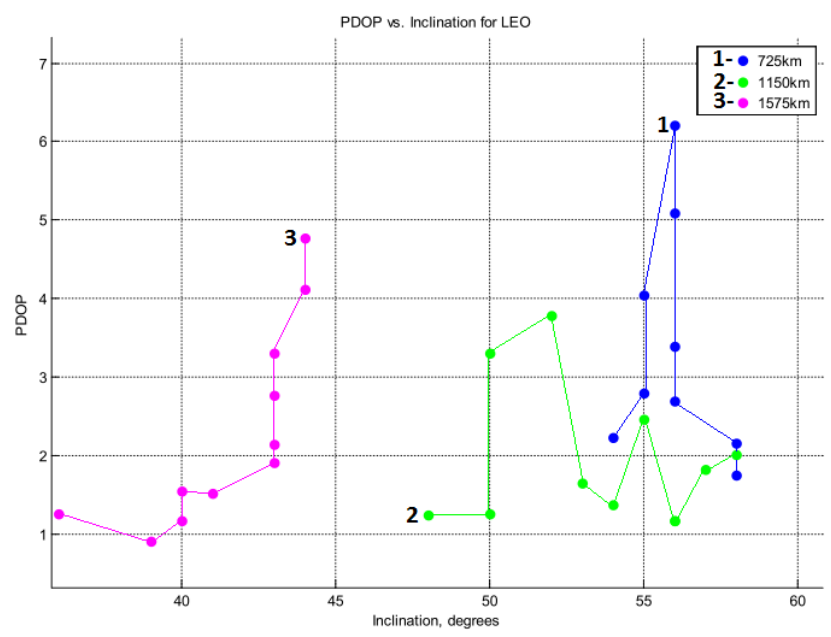

Figure 25: PDOP vs. Inclination for LEO
Since each altitude constellation possesses some solutions for the specific number of orbit planes and satellites per plane, which can be represented by the relationship between PDOP and the inclination of LEO constellations. Inclination is the only design parameter than could affect PDOP if the number of orbit planes and satellites per plane keep constant.

Figure 26 illustrates the design results of inclinations and PDOP values as solutions of the LEO constellations, in which number of orbit planes and satellites per plane are combined as group parameters. For some of the solutions, if the group parameter that number satellite per plane is larger than the number of orbit plane, the constellation may provide the solution with lower PDOP value, for example 11planes/13spp and 10planes/12spp cases. However, for equal number of orbit plane and number satellite per plane, the PDOP value maybe very large, such as 10planes/10spp case.

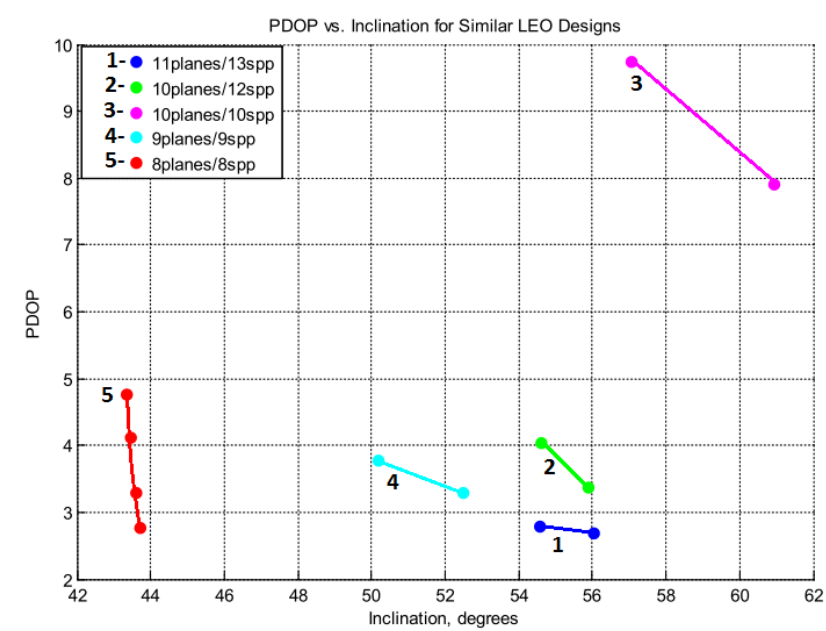

Figure 26: PDOP vs. Inclination for combined LEO parameters

The couple parameters analyzed in MEO and HEO design are number of orbit planes and PDOP values. The simulation results for different altitude of MEO and HEO constellation are shown in Figure 27. The 
results display that the number of orbit planes are increased along with smoothly decreased PDOP.

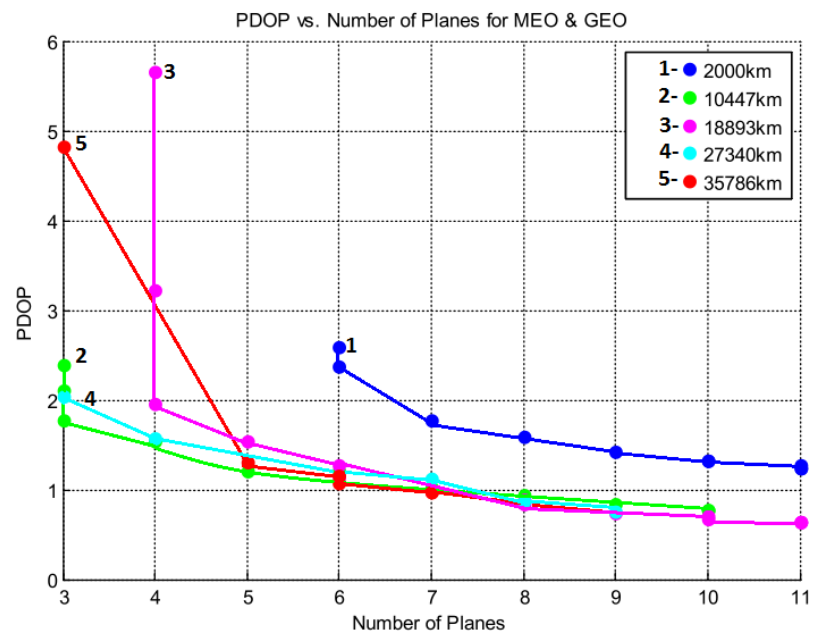

Figure 27: PDOP vs. Number of orbit Planes for MEO and GEO design

Figure 28 illustrates the relationship between number of satellites per plane and PDOP values in different MEO altitude constellations. It can be seen that the results are much different from the similar cases of the LEO constellations in Figure 23 as the trends are not smooth. With the same number satellites, different PDOP could require different number of orbit planes. For a PDOP value around one, the number of satellites decreases as the altitude increases from $10447 \mathrm{~km}$ to $18893 \mathrm{~km}$, but the number of satellite per plane for both altitudes are equal. This is consistent with the LEO results.

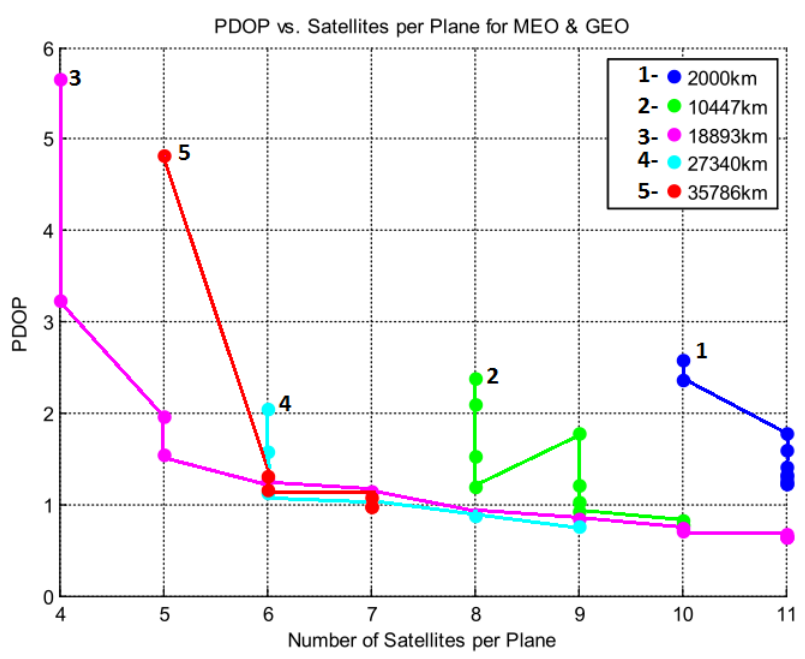

Figure 28: PDOP vs. Satellites per Plane for MEO

Figure 29 shows the relationship between number of satellites per plane and number of orbit planes for the MEO and HEO test cases. For each altitude, in pace with the number of orbit planes increases, the number of satellites per plane either remains constant or increases. The largest difference between two values is six as 3 planes/9spp. The first and second lowest altitude results do not illustrate much difference in number of satellites per plane when the number of orbit planes increase. This could be attributed to the lower altitude constellations requiring more satellites in each plane to produce PDOP values less than six. It is more evident when the altitudes increase. The variety of satellites per plane for the higher altitudes provides better coverage. Therefore, the number of satellites per plane does not remain at the upper bound values. The other dominating results illustrate that a roughly linear trend between the number of satellites per plane and the number of planes could be implemented by integrating different altitude constellations. 


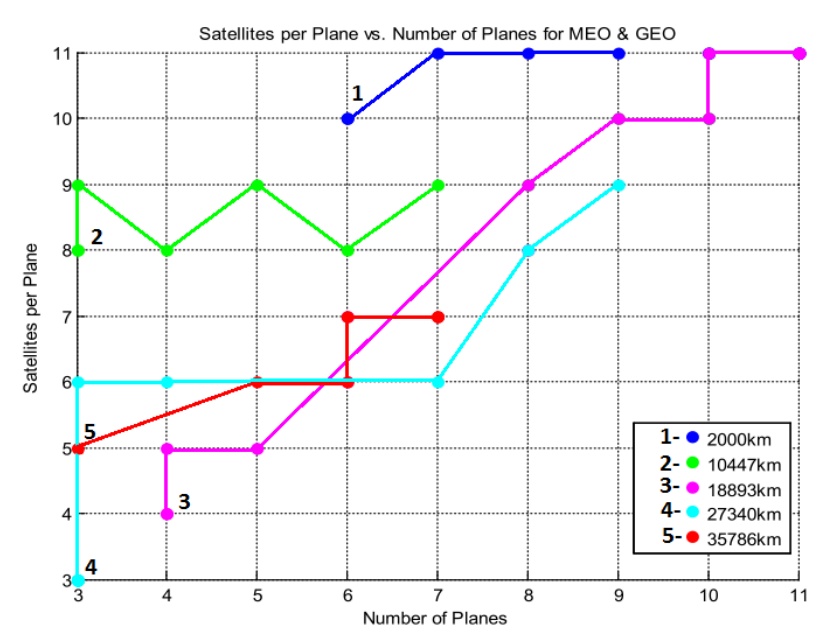

Figure 29: Satellites per Plane vs. Number of Planes for MEO

Equations (10) and (11) represent the respective trends between these parameters.

$s p p=0.2471 *$ planes +7.3817

where $\mathrm{R}^{2}=0.83$.

$$
s p p=0.7623 * \text { plane }+1.827
$$

where $\mathrm{R}^{2}=0.79$. Figure 30 shows relation between the inclination of different altitude MEO and the PDOP values. The first three altitudes demonstrate a 10 degree range of inclination, which matches the results of LEO in Figure 25. The higher altitude MEO and HEO do not illustrate the same range in inclination. Both altitude results are more sporadic, but stay within a 20 degree range. Ignoring the higher altitude MEO and HEO results, the performances of two lower altitude MEO in a larger inclination range are better than high altitude of $18893 \mathrm{~km}$ results. A similar trend can be seen in the LEO results. This makes sense that more inclination is needed at lower altitudes to make PDOP values less than six.

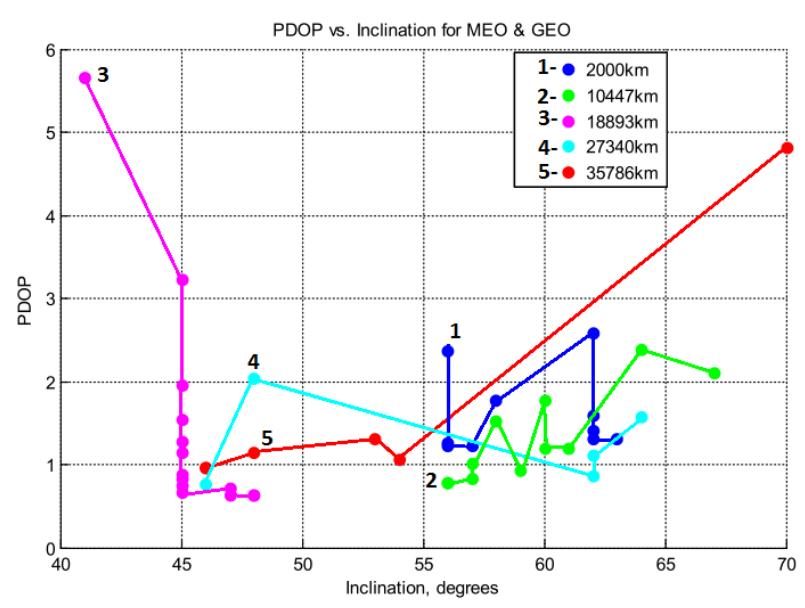

Figure 30: PDOP vs. Inclination for MEO

To analyze the effects on PDOP (Position solution Dilution of Precision) for different solutions with the same number of orbit planes and number of satellites per plane, Figure 31 shows the solutions for MEO and HEO with equal number of orbit planes and number of satellites per plane.

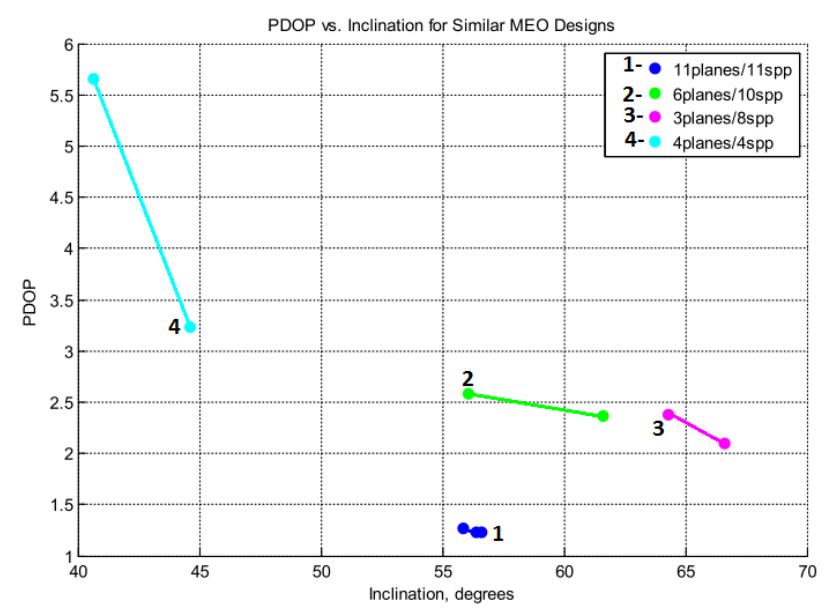

Figure 31: PDOP vs. Inclination for Similar MEO Designs

The higher altitude MEO and HEO do not have any solutions with equal orbit planes and satellites per plane. The results shown here are taken from the first three altitudes MEO. For each set of solutions, the inclination is slightly larger for a smaller PDOP value. This is consistent with results of the LEO in Figure 26. Although inclination do not demonstrate 
a clear increase or decrease as PDOP changing for the overall solutions, the similar solutions illustrate that it is possible to decrease PDOP with a larger inclination.

\section{Results}

The same examples are taken as the current GPS constellation, The concave portion of respective Pareto fronts in design results is used to analyze how PDOP (Position solution Dilution of Precision) value changes with the latitude. Figure 32 illustrates the PDOP values change with latitudes for both GPS and the optimal design of LEO constellations. The GPS constellation possesses lower PDOP values, and there are no major outliers. The optimal LEO design possesses PDOP values that are greater than 2 when latitude is less than -45 degrees or greater than 45 degrees. The LEO design shows increase of PDOP at latitudes less than -30 degrees or greater than 30 degrees. The GPS constellation shows the opposite trend for those latitudes as it decreases at latitude less than -30 degrees or greater than 30 degrees. Since the GPS constellation is at a higher altitude, it is able to provide better geometry globe coverage especially at higher latitudes.

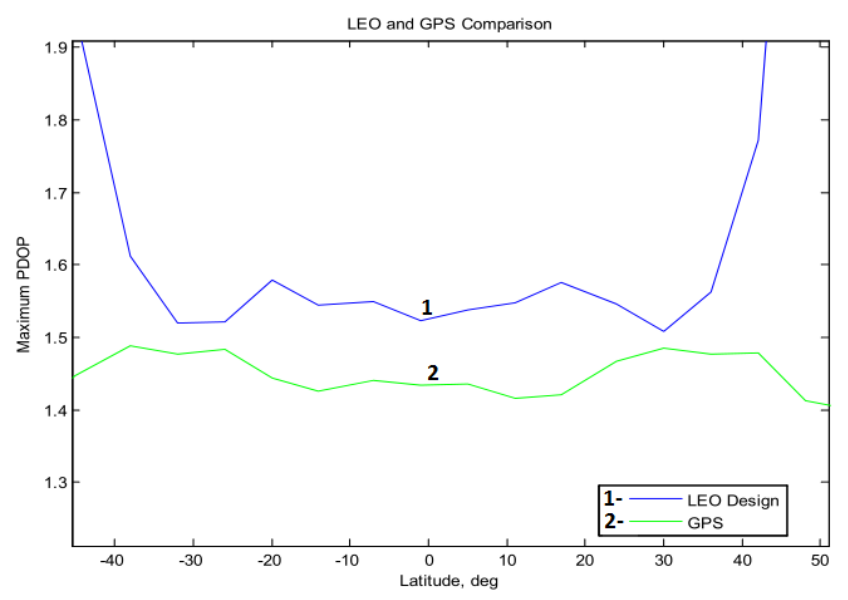

Figure 32: LEO and GPS Comparison
Figure 33 shows the comparison of the optimal design for MEO and the GPS with varying of PDOP values against the latitude changes. The MEO design produced lower values of PDOP for all latitudes.

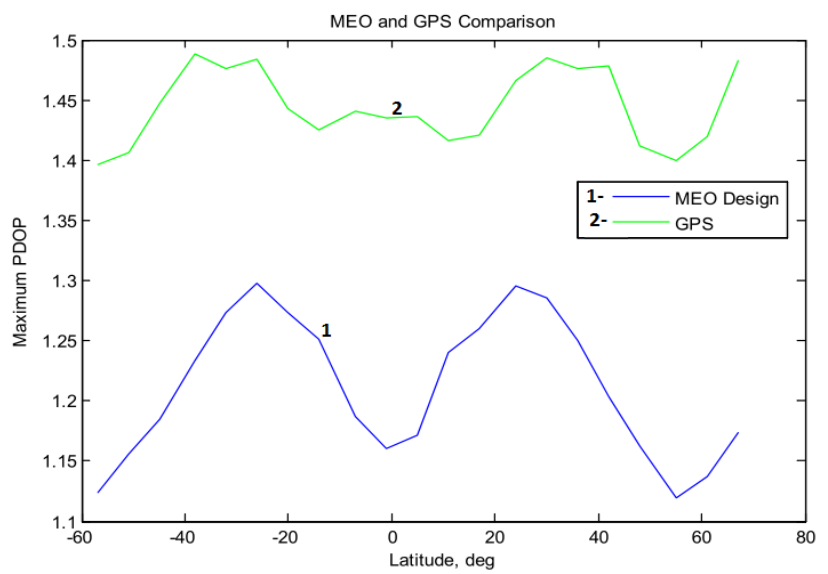

Figure 33: MEO and GPS Comparison

The MEO altitude is approximately $10,000 \mathrm{~km}$ lower than the GPS and possesses 21 extra satellites. Both constellations show peaks at around latitude of -30 degrees and 30 degrees. This is because of both constellations have similar inclinations (around 55 degrees). The MEO design shows lower value of PDOP at latitude of 0 degrees for the reason that it is difficult to have multiple satellites in view over the equator for inclined constellations.

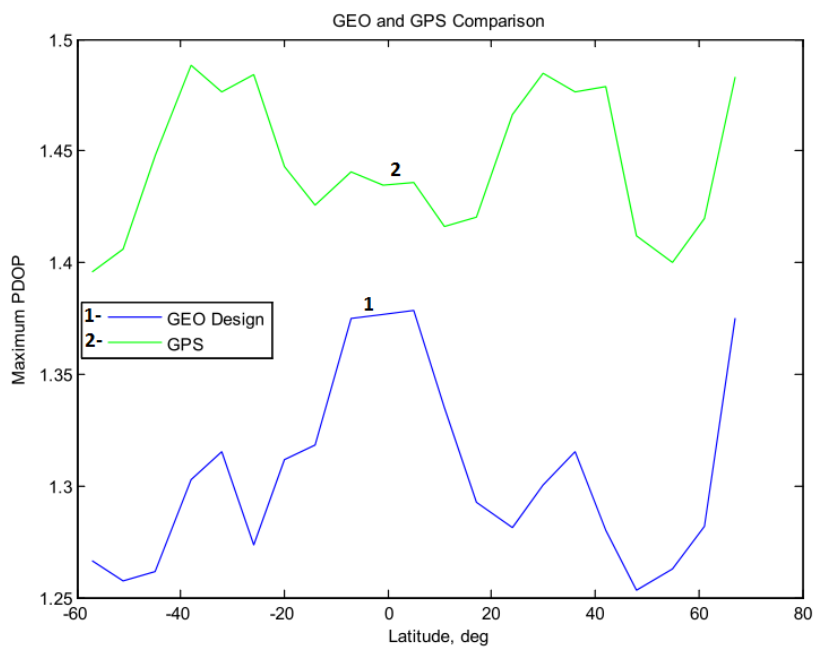

Figure 34: GEO and GPS Comparison 
Figure 34 illustrates the comparison of deigned HEO and GPS constellations. The designed HEO produces lower PDOP values than GPS constellation with excluding latitudes less than -60 degrees or greater than 60 degrees. Both PDOP values drop to the valley at 50 degree latitude. For designed HEO, the peak values of PDOP occur around -7 to 5 degree latitudes. However, the PDOP values of GPS at latitude -7 to 5 degrees are lower than the others. Both constellations maintain PDOP values less than six for all latitudes.

The MOGA is not applied in the current GPS constellation and optimal designed constellation. The results of this paper are obtained by a smaller range between the lower and upper bounds of the design parameters used for promoting GPS case. As supposed and defined early, the main advantage of utilizing the MOGA is determining the RAAN increment, inclination, and the relationship between number of orbit planes and satellites per plane. The altitude and transmit power are kept relatively constant, so the design space is mostly consisted of the number of orbit planes, satellites per plane, RAAN increment, and inclination. The number of orbit planes and satellites per plane illustrate an obvious relationship between PDOP and cost. However, the inclination and RAAN increments are more random and do not illustrate an obvious relationship. Thus, it would be difficult to directly determine points on the Pareto front when these parameters are varying. The MOGA would provide more of an advantage if the other design variables are analyzed with respect to this problem because the design space would increase.

For each altitude constellation design, parameter sweep can be completed by searching optimal number of orbit planes and satellites per plane. A point of the Pareto fronts can be selected by keeping one of the parameters constant and varying the other. Figure 35 illustrates the parameter sweep for the number of orbit planes using the LEO 3 test case. As the number of orbit planes varying, the points remain relatively close to the Pareto front. The points generated by the parameter sweep do not result in a better PDOP for a lower cost.

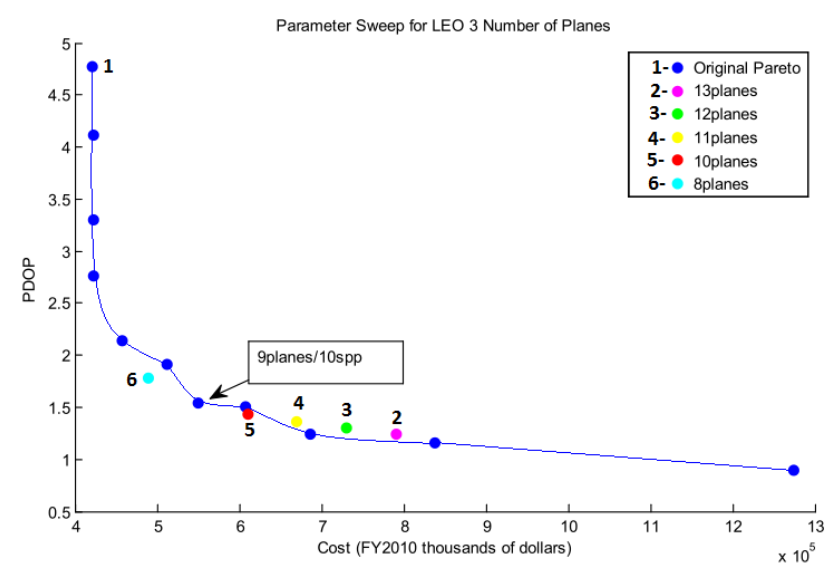

Figure 35: Parameter Sweep for LEO 3 Number of Orbit Planes Is PDOP

Figure 36 illustrates a parameter sweep of the satellites per plane in the LEO 3 test case. The points generated from the parameter sweep remain close to the Pareto front. Both Figure 35 and Figure 36 show an intense relationship between the number of orbit planes and satellites per plane. 


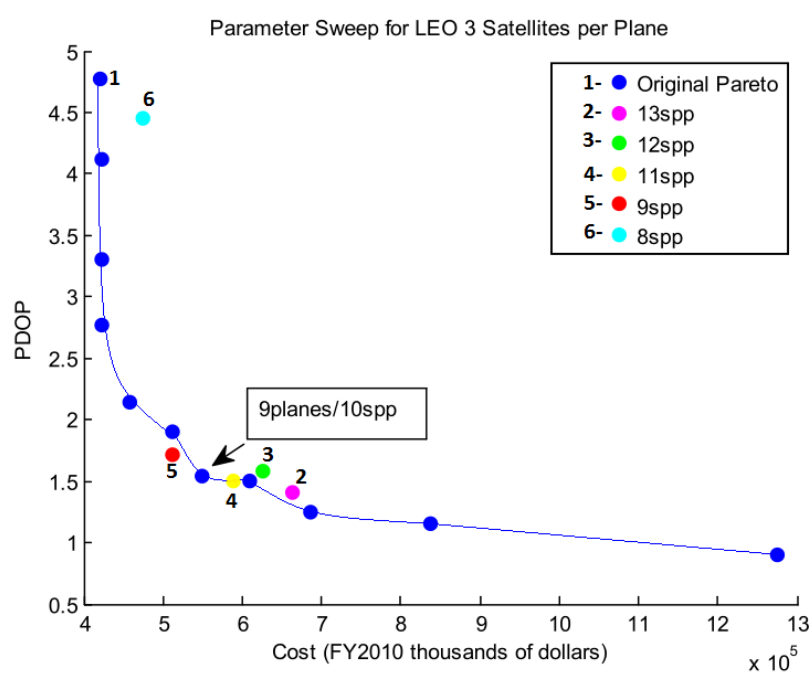

Figure 36: Parameter Sweep for LEO 3 Satellites per Plane

By varying these parameters, additional points are determined; this is a result of using a small generation number for the simulations. If a larger generation number are used, it is possible that the MOGA would determine these points as well. Figure 37 illustrates the parameter sweep for the number of orbit planes in the MEO 2 test case. These results are similar to the LEO altitude test case. Most of the points generated from the parameter sweep fall in among between points on the Pareto front.

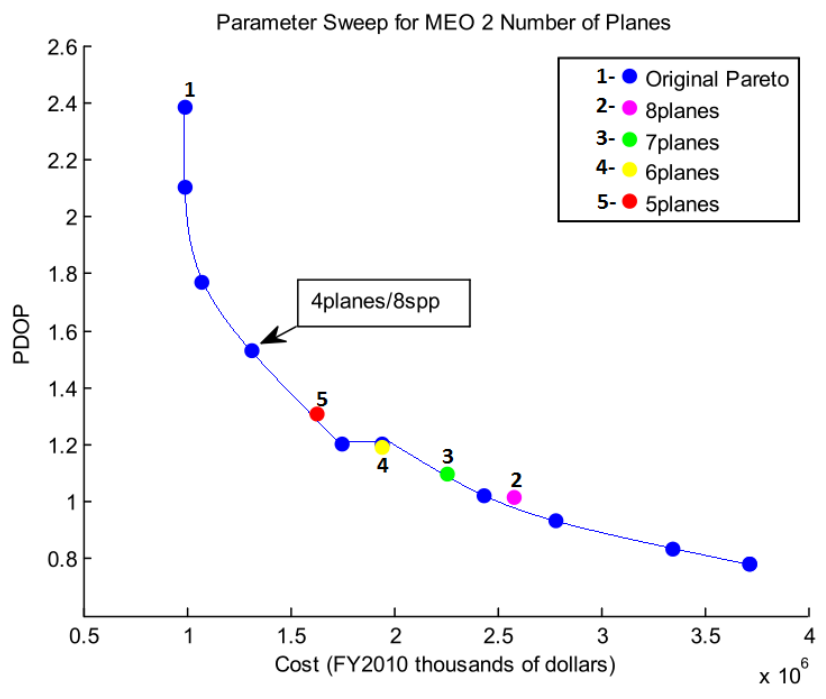

Figure 37: Parameter Sweep for MEO 2 Number of Orbit

Planes
In Figure 38 the number of satellites per plane was varied for the MEO 2 test case. The parameter sweep produced a smooth curve along Pareto front points as the previous results. The relationship between PDOP and cost remains the same as the number of satellites per plane varies.

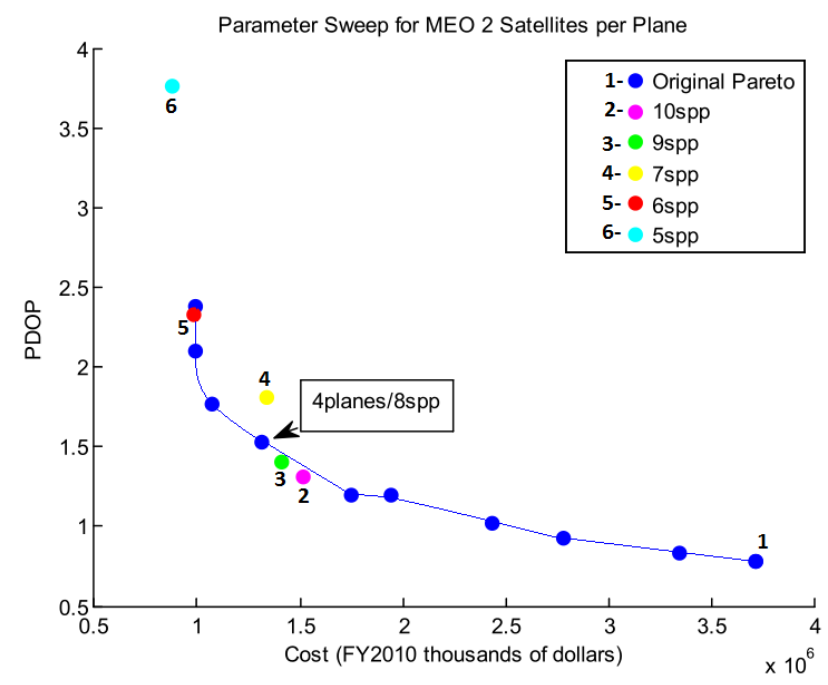

Figure 38: Parameter Sweep for MEO 2 Satellites per Plane

Figure 39 shows the parameter sweep for the number of orbit planes in the GEO test case. The parameter sweep generated points among the points of the Pareto front. This is also a result of the low generation number used for the simulations. The point produces by six planes is almost exactly equal to one of the points of the Pareto front.

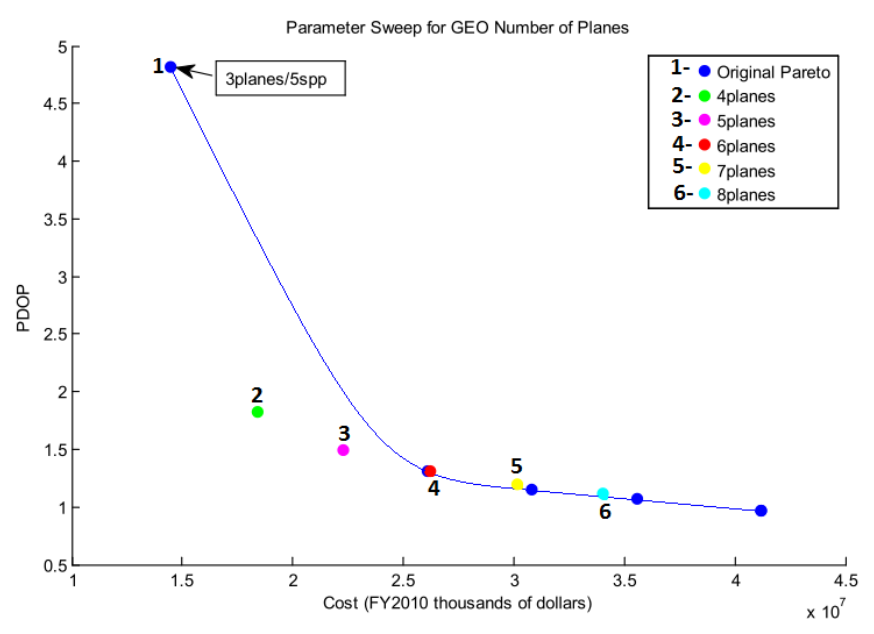

Figure 39: Parameter Sweep for GEO Number of Orbit Planes 
Figure 40 illustrates number of satellite per plane in parameter sweep for the HEO test case. These results are similar to above two altitude cases. All parameter sweeps show that it is possible to determine the points close to Pareto front generated by the MOGA. None of the points generated by the parameter sweeps can produce better PDOP (Position solution Dilution of Precision) values for a lower cost. Utilizing the MOGA for this problem is benefit for determining the relationship between the number of orbit planes and the number of satellites per plane. The MOGA is also benefit for determining the range of inclinations that are associated with the different orbital altitudes. Understanding these trends will allow for further research into the constellation design. Whether increasing the design space and continuing to use the MOGA for optimization or using a different optimization method, the trends and relationships will be necessary and determined from these results.

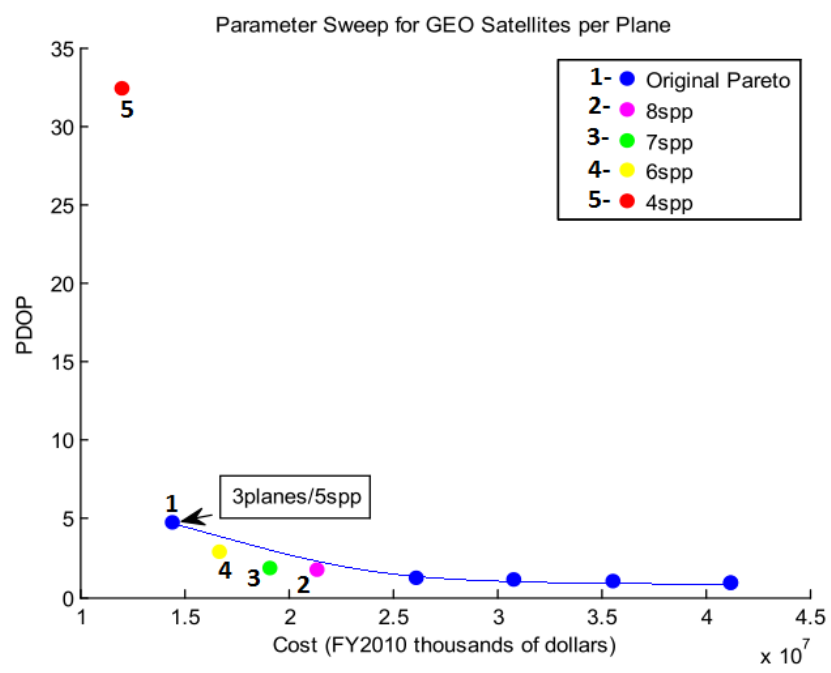

Figure 40: Parameter Sweep for HEO Satellites per Plane

\section{Conclusions and further discussions}

This paper results demonstrate using the tools of MATLAB, MOGA and STK in the optimization of constellation designs. There are several limitations in the simulations of optimal constellations. There are eleven design parameters used in the simulation, and four of them as the eccentricity, argument of perigee, RAAN, and mean anomaly are set to zero. Therefore, the effects of these parameters on a constellation system at different altitudes are omitted. There is not discussion of the difference in performance between circular orbits and elliptical orbits either. The median of the optimal PDOP values is used to determine the central tendency of PDOP values across the latitudes. There is no distribution in PDOP values that are input to the MOGA. Although threshold could be easily implemented, extensive sweeps are outside the scope of this work. The variation of PDOP over latitude is shown in several constellation designs, but the specific trend of PDOP values for all altitudes is not analyzed. Transmit power is calculated specifically for each altitude to ensure the proper size of satellite and link closure. Therefore, the MOGA do not have the option of selecting cheaper satellites for higher altitude orbits.

The spacecraft cost increases as the altitude increasing to account for more transmit power required. This limits the options for spacecraft design of constellations at higher altitudes. Walker constellations are taken as example in this thesis, so all the satellites in a constellation are the same size. As consequence, the performance and cost of constellations are not analyzed by mixed size satellites. This paper presented possible constellation designs at different orbital altitudes. As expected, the PDOP values are improved by 
designing higher altitude constellations, since the required number of satellites decreases as altitude increases. The cost of the design results varies along with the altitude, but in general, the LEO design results illustrate less expensive options. Several of the design results are compared to the GPS constellation, and both the MEO and HEO constellation designs maintain PDOP values less than the GPS system.

References:

[1] J. Wertz, D. Everett and J. Puschell, "Space Mission Engineering": The New SMAD, Hawthorne: Microcosm Press, 2011.

[2] Daniel Casanova, Martin Avendano, Daniele Mortari "Optimizing Flower Constellations for Global Coverage" AIAA/AAS Astrodynamics Specialist Conference, 2012, 10.2514/6.20124805.

[3] Ferringer, M.P., Spencer, D.B., "Satellite Constellation Design Tradeoffs Using MultipleObjective Evolutionary Computation", Journal of Spacecraft and Rockets. Vol. 43, no. 6, pp. 14041411. Nov.-Dec. 2006

[4] 1968 (10.7MB PDF) - NASA ,"Saturn V Flight Systems Analysis",Saturn V Launch Vehicle Emergency Detection System Analysis, SA504.The BoeingCompany Space Division Launch Systems Branch, 1968.www.nasa.gov >centers > pdf > 744319main_1968[41]Book, Miller, D. E., and Sedwick, R. J., "Grand Challenges in SpaceTechnology: Distributed Satellite Systems",
U.S. Air Force ResearchLaboratory, Cambridge, MA, 2001, pp. 1-15.

[5] General William L. Shelton," "Resiliency and Disaggregated Space Architectures," U.S. Air ForceSpace Command, 2013, https://fas.org/spp/military/resiliency.pdf.

[6] Shelton, W. L., "Presentation to Subcommittee on Strategic Forces,Senate Armed Services Committee, United States Senate," 2013, https://www.hsdl.org/?view\&did=746029.

[7] F. Vatalaro, G. Corazza, C. Caini and C. Ferrarelli, "Analysis of LEO, MEO, and GEO Global Mobile Satellite Systems in the Presence of Interference and Fading," IEEE Journal on Selected Areas in Communications, vol. 13, no. 2, p. 10, 1995.

[8] E. D. Kaplan and C. J. Hegarty, Understanding GPS: Principles and Applications, Norwood: Artech House, Inc, 2006.

[9] P.Przyborski, "NASA Earth Observatory," NASA, [Online]. Available: http://earthobservatory.nasa.gov/Features/OrbitsC atalog/page2. [Accessed 10 January 2015].

[10] Bate, Mueller and White, "Fundamentals of Astrodynamics", Dover Publications, 1971.

[11] J. Sellers, “Understanding Space: An Introduction to Astronautics", New York: McGraw-Hill Higher Education, 2005.

[12] "STK 10 Help," AGI, [Online]. Available: http://www.agi.com/resources/help/online/stk/10. 
1/index.html?page=source $\% 2$ Fstk $\% 2 . h t m$.

[Accessed 10 January 2015].

[13] Carrasquillo, R. L., and Bertotto, D., "ECLSS Design for the International Space Station Nodes 2 \& 3," SAE Technical Paper No. 1999-01-2146, Society of Automotive Engineers, Warrendale, PA, 29th ICES (International Conference on Environmental Systems), 1999.

[14] Williams, M., "Falcon Heavy Vs. Saturn V," Universe Today, Aug. 2, 2016,

https://www.universetoday.com/129989/saturn-vvs-falcon heavy/, accessed Jan. 5, 2018.

[15] R. M. Alkan, H. Karaman and M. Sahin, "GPS, Galileo, and GLONASS Satellite Navigation Systems and GPS Modernization," in Recent Advances in Space Technologies, 2nd International Conference on, pgs.390-394, 2005.

[16] Jones, H. W., "Would Current International Space Station (ISS) Recycling Life Support Systems Save Mass on a Mars Transit?" ICES 2017-85, 47th International Conference on Environmental Systems, 2017.

[17] Jones, H. W., "Oxygen Storage Tank Systems for Mars Transit," ICES 2017-89, 47th International Conference onEnvironmental Systems, 2017.

[18] Greg, "SpaceX - Low cost access to space." Harvard Business School, Technology and Operations Management, Posted on December 9, 2015, https://rctom.hbs.org/submission/spacexlow-cost-access-to-space/, accessed Jan. 8, 2018.
[19] MATLAB Optimization Toolbox User's Guide, The MathWorks, Inc.,Natick, MA, 2013, pp. 1160.

[20] "Getting Started with CPLEX for MATLAB," IBM ILOG CPLEXOptimization Studio V12.6.1 Documentation, Armonk, NY, March 2015,pp. 112 , http://www 01.ibm.com/support/knowledgecenter/SSSA5P_1 2.6.1/ [retrieved Feb. 2016].

[21] Zimmerman, R., "The Actual Cost to Launch," Behind the Black, April 6, 2012, http://behindtheblack.com/behindtheblack/essays-and-commentaries/the-actualcost-to-launch/, accessed Jan. 8, 2018.

[22] Gruss, M., "Disaggregation Gets Traction in 2015 Pentagon Budget Request,” Space News, 7 March 2015, http://spacenews.com/39773disaggregationgets-traction-in-2015-pentagon-budget-request/ [retrieved 2017].

[23] CHEN Ruming, "Design and Analysis of nongeostationary orbit communication system. Modem Science Technology of Telecommunications," Journal of Electronics and Information Technology,2004,(1):40-44(in chinese)

[24] Collopy, Paul D. and Horton, Randolph R. "Value Modeling for Technology Evaluation." AIAA Paper 2002-3622, American Institute of Aeronautics and Astronautics, Reston, VA, 2002.

[25] Dyer, J. S. (2016). Multiattribute utility theory (MAUT). International Series in Operations 
Research and Management Science, 233, 285314. DOI: 10.1007/978-1-4939-3094-4_8.

[26] Cook, Harry E. Product Management. Chapman \& Hall, London, 1997.

[27] Optimal Earth orbiting satellite constellations via a Pareto Genetic Algorithm, William Mason, Victoria Coverstone-Carroll, John Hartmann,AIAA/AAS Astrodynamics Specialist Conference and Exhibit, 1998, 10.2514/6.19984381

[28] Orbit Design for Ground Surveillance Using Genetic Algorithms, Ossama Omarabdelkhalik, Daniele Mortari, Journal of Guidance, Control, and Dynamics, 2006, Vol.29: 1231-1235, $10.2514 / 1.16722$

[29] Low Earth Orbit Debris Removal Technology Assessment Using Genetic Algorithms, Matthew L. Marcus, Raymond J. Sedwick, Journal of Spacecraft and Rockets, 2017, Vol.54: 1110$1126,10.2514 / 1 . \mathrm{A} 33671$

[30] Low Earth Orbit Debris Removal Technology Assessment Using Genetic Algorithms, Matthew L. Marcus, Raymond J. Sedwick, Journal of Spacecraft and Rockets, 2017, Vol.54: 11101126, 10.2514/1.A33671.

[31] I. Budianto and J. Olds, "Design and Deployment of a Satellite Constellation Using Collaborative Optimization," Journal of Spacecraft and Rockets, vol. 41, no. 6, pp. 956-963, 2004.
[32] Dufour, F., Lasserre, E., and Brousse, P., “A Multistage Approach to Design and Optimize a Communication Satellite Constellation," IAF-99A.2.09, 50th International Astronautical Congress, Amsterdam, The Netherlands, Oct. 4-8, 1999.

[33] Javad Alishah1, Soroush Maddah1, Javad Alinejad2,1 and Yasser Rostamiyan1" 3D numerical simulation of flap geometry optimization around the cylinder to collection of split up droplet" https://doi.org/10.1088/1873-7005/ac13bc

J. Alinejad \& Mohammad Mohsen Peiravi," Numerical analysis of secondary droplets characteristics due to drop impacting on 3D cylinders considering dynamic contact angle" https://link.springer.com/article/10.1007/s1 1012-020-01240-Z

[35] J. Alinejad \& J. A. Esfahani," Lattice Boltzmann simulation of 3-dimensional natural convection heat transfer of $\mathrm{CuO} /$ water nanofluids" https://link.springer.com/article/10.1134/S 0869864317010103

\section{Creative Commons Attribution License 4.0} (Attribution 4.0 International, CC BY 4.0)

This article is published under the terms of the Creative Commons Attribution License 4.0

https://creativecommons.org/licenses/by/4.0/deed.en_US 